\title{
Structure theory of finite conformal algebras
}

\author{
by
}

\author{
Alessandro D'Andrea
}

Laurea in Matematica, Università degli Studi di Pisa (1994)

Diploma, Scuola Normale Superiore (1994)

Submitted to the Department of Mathematics

in partial fulfillment of the requirements for the degree of

Doctor of Philosophy

at the

MASSACHUSETTS INSTITUTE OF TECHNOLOGY

June 1998

(C)Alessandro D'Andrea, 1998. All rights reserved.

The author hereby grants to MIT permission to reproduce and to distribute publicly paper and electronic copies of this thesis document in whole or in part.

Author

Department of Mathematics May 6, 1998

Certified by .

Victor G. Kac Professor of Mathematics Thesis Supervisor

Accepted by

Richard B. Melrose

WASSACHUSETTS WES IW IA

OF TECHAOLO = "

Chairman, Department Committee

JUN 011998

LIBRARIES 


\title{
Structure theory of finite conformal algebras
}

\author{
by \\ Alessandro D'Andrea
Submitted to the Department of Mathematics on May 6, 1998, in partial fulfillment of the requirements for the degree of \\ Doctor of Philosophy
}

\begin{abstract}
In this thesis I gave a classification of simple and semi-simple conformal algebras of finite rank, and studied their representation theory, trying to prove or disprove the analogue of the classical Lie algebra representation theory results. I re-expressed the operator product expansion (OPE) of two formal distributions by means of a generating series which I call " $\lambda$-bracket" and studied the properties of the resulting algebraic structure. The above classification describes finite systems of pairwise local fields closed under the OPE.
\end{abstract}

Thesis Supervisor: Victor G. Kac

Title: Professor of Mathematics 


\section{Acknowledgments}

The few people I would like to thank are those who delayed my thesis the most. Even though I might have been able to finish my thesis earlier, my time at MIT would also have been much less pleasant without these people, with whom I shared joy and sadness, interest and idleness, relaxation and stress.

And although putting up with me as a roommate, playing a terrible bridge hand without blaming the awful contract on me, lending me books and CDs without expecting me to give them back, sending me birthday cards, hiding a huge aversion towards the most intolerable aspects of my character with a smile, sharing a trip in a van on a road to nowhere, sipping tea with me while listening to Schubert, helping me explore Boston at night, or giving me a call in the moment I need it the most, may all appear as the most ordinary abilities (and they are not), I know that I could never have survived the past four years without them.

Finally, my everlasting gratitude goes to my mother and my sister, who have always supported me despite the difficulties in understanding many of my inexplicable idiosyncrasies. 


\section{Contents}

1 Introduction $\quad 5$

2 Basic definitions $\quad 8$

3 Conformal linear algebra $\quad$ • 14

4 The annihilation algebra $\quad 23$

$\begin{array}{lll}5 & \text { Simple conformal algebras } & 30\end{array}$

$\begin{array}{lll}6 & \text { The Killing form } & 36\end{array}$

7 Semi-simple conformal algebras $\quad 43$

$\begin{array}{lll}8 & \text { Representation theory } & 47\end{array}$

9 A generalization: the case of several indeterminates 66 


\section{Chapter 1}

\section{Introduction}

In this thesis I am going to develop a structure theory of finite rank conformal algebras. The conformal algebra structure is an axiomatic description $[\mathrm{K}]$ of the operator product expansion (OPE) of chiral fields in a conformal field theory [BPZ]; in other words a conformal algebra is a Vertex algebra without the normally ordered product. The theory of conformal algebras of finite rank classifies finite families of pairwise local fields which are closed under the OPE, is the sense that only linear combinations of the generating fields and their derivatives may occur in the OPE of two fields.

On the other hand, the category of finite conformal algebras is (more or less) equivalent to the category of infinite-dimensional Lie algebras spanned by Fourier coefficients of a finite number of pairwise local fields (or rather formal distributions) that are closed under the OPE [K],[K3]. Hence the theory of finite conformal algebras provides a classification of these finite "formal distribution Lie algebras".

The main idea is to develop a structure theory of finite conformal algebras which is parallel to that of finite-dimensional Lie algebras through the interplay of formal distribution Lie algebras with their "annihilation algebras". As the latter are filtered

Lie algebras in the sense of E. Cartan, we can use the well developed techniques of Cartan's theory.

Using the powerful Cartan-Guillemin theorem [G] (see also [B]) and a conformal analogue of the Killing form, we obtain a classification of finite simple and semi-simple conformal algebras (Theorems 5.1 and 7.1). We also prove conformal analogues of 
the Lie theorem (Theorem 8.4) and of the Cartan-Jacobson theorem (Theorem 8.6) using a result on filtered Lie algebras from [CK].

Some of the conformal analogues of the finite-dimensional Lie algebra theory fail, however. For example, it is not true that a semi-simple conformal algebra is a direct sum of simple ones (cf. Theorem 7.1). The conformal analogues of the Levi theorem and the Weyl complete reducibility theorem fail as well (see $[\mathrm{KW}],[\mathrm{BKV}],[\mathrm{CKW}]$ ). This reflects the fact that the cohomology of simple conformal algebras [BKV] with non-trivial coefficients is highly non-trivial.

Another new important feature of conformal algebra theory is the fact that the conformal analogue of $\mathfrak{g l}_{N}$, which we call the general conformal algebra and denote by $g c_{N}$, is not finite. It is actually the conformal algebra associated to the Lie algebra of regular $N \times N$-matrix valued differential operators on $\mathbb{C}^{\times}$(Example 4.3); its central extension, denoted by $W_{1+\infty}^{N}$, plays an important role in physics. In this paper we develop the relevant conformal linear algebra.

The language of conformal linear algebra allows for the construction of the "conformal" equivalent of all standard algebraic structures. In a sense, the conformal algebras presented in this thesis are just the conformal version of ordinary Lie algebras. Associative conformal algebras describe the algebraic properties of conformal linear maps under composition and prove useful in the description of representation theory.

There are clear generalizations of the conformal algebra structure to the case of several indeterminates. Most of the theory stays unchanged, and the only major difference is that all indices become multi-indices. The same classification of finite rank conformal algebras should hold on to the case of several indeterminates, with algebra corresponding to simple Cartan-type Lie algebras and their affinizations showing up as the basic blocks of semi-simple conformal algebras.

This thesis is structured as follows: in Chapter 2 we describe the basic questions we want to answer. We introduce systems of pairwise local formal distributions, and describe their algebraic structure in terms of the ${ }_{(n)}$ products, thus producing the definition of a conformal algebra. In Chapter 3 we define the concepts of conformal 
linear map and of $\lambda$-product. The defining axioms for a conformal algebra are reexpressed in term of the $\lambda$-product, allowing us to prove a few results about the torsion submodule of a finitely generated (as $\mathbb{C}[\partial]$-module) conformal algebra. A classification of conformal algebra structures for free modules of rank one is also given.

In Chapter 4 we define the central notions of annihilation algebra and extended annihilation algebra, along with their completions. We show how algebraic properties of a conformal algebra are often reflected at the level of its annihilation algebra. In Chapter 5 we show (Proposition 5.1) that the extended annihilation algebra completely characterizes a torsionless conformal algebra, and we give a classification of finite simple conformal algebras.

In Chapters 6 and 7 we give a classification of semi-simple conformal algebras. We construct a "Killing form" and we show that the orthogonal subspace to an ideal is a subalgebra, but not necessarily an ideal. Therefore every semi-simple conformal algebra can be expressed as a semi-direct (but not necessarily direct) sum of simple algebras.

In Chapter 8 we give a survey of the basic results in representation theory of conformal algebras. The final theorem of the chapter is the conformal version of Cartan-Jacobson theorem, the proof of which involves many of the techniques we have developed in the rest of the thesis. Finally in Chapter 9 we outline the present state of research on conformal algebras in several indeterminates. A tentative classification of finite simple conformal algebras in several indeterminates is given. Most of this thesis originates from a joint work with my advisor [DK].

Unless otherwise specified, all vector spaces, linear maps and tensor products are considered over the field of complex numbers $\mathbb{C} . \mathbb{Z}_{+}$will denote the set of non-negative integers. 


\section{Chapter 2}

\section{Basic definitions}

We start by defining the basic objects we want to consider. Let $V$ be a (possibly infinite-dimensional) vector space. A formal distribution with values in $V$ is a power series of the form $a(z)=\sum_{n \in \mathbb{Z}} a_{n} z^{-n-1}$ where $a_{n} \in V$. The vector space of these series is denoted by $V\left[\left[z, z^{-1}\right]\right]$. Such series are called formal distributions since we have a standard $V$-valued pairing with $\mathbb{C}\left[z, z^{-1}\right]$ which is given by

$$
\langle a, p\rangle=\operatorname{res}_{z} a(z) p(z)
$$

where $\operatorname{res}_{z} a(z)=a_{0}$ is the coefficient of $z^{-1}$ and $p$ is a Laurent polynomial in $z$. Of course we have $a_{n}=\left\langle a, z^{n}\right\rangle$. Vectors $a_{n}$ are also called Fourier coefficients of $a(z)$ and completely determine it. A formal distribution $a(z, w)$ in two variables is similarly defined as a series of the form $\sum_{m, n \in \mathbb{Z}} a_{m, n} z^{-m-1} w^{-n-1}$, and the space of these series is denoted by $V\left[\left[z, z^{-1}, w, w^{-1}\right]\right]$.

Suppose now we have vector spaces $U, V, W$. Any linear map $\alpha: U \otimes V \rightarrow W$ induces a map

$$
\tilde{\alpha}: U\left[\left[z, z^{-1}\right]\right] \otimes V\left[\left[w, w^{-1}\right]\right] \rightarrow W\left[\left[z, z^{-1}, w, w^{-1}\right]\right] .
$$

If $u \in U\left[\left[z, z^{-1}\right]\right], v \in V\left[\left[w, w^{-1}\right]\right]$ are formal distributions, the pair $(u, v)$ is called a 
local pair whenever for some $N \in \mathbb{Z}_{+}$:

$$
(z-w)^{N} \tilde{\alpha}(u(z) \otimes v(w))=0 .
$$

Let $\mathfrak{g}$ be a Lie algebra, and let $a, b$ be $\mathfrak{g}$-valued formal distributions. If we choose our bilinear map $\alpha$ to be the Lie bracket $[]:, \mathfrak{g} \otimes \mathfrak{g} \rightarrow \mathfrak{g}$, then $(a, b)$ is a local pair if for some $N \in \mathbb{Z}_{+}$: .

$$
(z-w)^{N}[a(z), b(w)]=0
$$

where we denoted $[\widetilde{[,]}(a(z) \otimes b(w))$ by $[a(z), b(w)]$. It is clear that in this case $(b, a)$ is also a local pair: we will say $a$ and $b$ are mutually local. When considering locality of formal distributions with values in a Lie algebra, we will always assume it is with respect to $[$,$] .$

Definition 2.1. Let $\mathfrak{g}$ be a Lie algebra, and $\mathcal{F}$ a family of mutually local formal distributions with values in $\mathfrak{g}$. The pair $(\mathfrak{g}, \mathcal{F})$ is a formal distribution Lie algebra if $\mathfrak{g}$ is spanned by Fourier coefficients of formal distributions from $\mathcal{F}$.

Remark 2.1. Every Lie algebra can be trivially made into a formal distribution Lie algebra by choosing $\mathcal{F}=\{g(z) \mid g \in \mathfrak{g}\}$ where $g(z)=\sum_{n \in \mathbb{Z}_{+}} g z^{-n-1}$, but we will be mostly concerned with Lie algebras that are spanned by Fourier coefficients of a finite number of formal distributions. Also, this formal distribution Lie algebra is not maximal (see Definition 2.4 below).

The Dirac delta distribution is the $\mathbb{C}$-valued formal distribution

$$
\delta(z, w)=\sum_{n \in \mathbb{Z}} z^{n} w^{-n-1}
$$

It will often be denoted by $\delta(z-w)$. This notation is consistent with taking derivatives since $\partial_{z} \delta(z-w)=-\partial_{w} \delta(z-w)$.

The delta distribution enjoys the property $(z-w) \delta(z-w)=0$. In general we shall have $(z-w)^{m} \partial_{w}^{n} \delta(z-w)=0$ if $m>n$. The converse is also true in some sense. If $a(z, w)$ is a distribution such that $(z-w)^{N} a(z, w)=0$, then there exist formal 
distributions $a^{n}(w)$ such that $[\mathrm{K}]$ :

$$
a(z, w)=\sum_{n=0}^{N-1} a^{n}(w) \partial_{w}^{(n)} \delta(z-w)
$$

Here and further we use notation $x^{(n)}=x^{n} / n$ !. Furthermore, the $a^{n}$ are uniquely determined:

$$
a^{n}(w)=\operatorname{res}_{z}(z-w)^{n} a(z, w) .
$$

In this way we know that if $(a, b)$ is a local pair, we can then find formal distributions denoted by $\left(a_{(n)} b\right)(w)$ such that

$$
\tilde{\alpha}(a(z) \otimes b(w))=\sum_{n=0}^{N-1}\left(a_{(n)} b\right)(w) \partial_{w}^{(n)} \delta(z-w)
$$

The formal distribution $a_{(n)} b$ is $\mathbb{C}$-bilinear in $a$ and $b$ and it is called the $n$-th product of $a$ and $b$. It is not generally $\mathbb{C}[\partial]$-bilinear in $a$ and $b$, as from (2.3) we have:

$$
(\partial a)_{(n)} b=-n a_{(n-1)} b, \quad a_{(n)} \partial b=\partial\left(a_{(n)} b\right)+n a_{(n-1)} b
$$

In particular, $\partial$ is a derivation of all $n$-th products $a_{(n)} b$.

Definition 2.2. Let $\mathfrak{g}$ be a Lie algebra. A space $\mathcal{F}$ of $\mathfrak{g}$-valued formal distributions in $z$ is called a conformal family if it is closed under derivative $\partial_{z}$ and all the bilinear $\operatorname{products}_{(n)}$ just defined.

One knows $[\mathrm{K}]$ that if $(\mathfrak{g}, \mathcal{F})$ is a formal distribution Lie algebra we can always include $\mathcal{F}$ in the minimal conformal family $\overline{\mathcal{F}}$. Note that $\overline{\mathcal{F}}$ can be viewed as a $\mathbb{C}[\partial]-$ module, where the action of $\partial$ is given by $(\partial a)(z)=\partial_{z} a(z)$. The formal distribution Lie algebra is called finite if $\overline{\mathcal{F}}$ is a finitely generated $\mathbb{C}[\partial]$-module.

Proposition 2.1. [K] The Lie algebra axioms for $\mathfrak{g}$ and the very definition of $a_{(n)} b$ translate into the following properties:

(C1) $a_{(n)} b=0$ for $n>>0$, 
(C2) $(\partial a)_{(n)} b=-n a_{(n-1)} b, \quad a_{(n)} \partial b=\partial\left(a_{(n)} b\right)+n a_{(n-1)} b$,

(C3) $a_{(n)} b=-\sum_{j=0}^{\infty}(-1)^{n+\jmath} \partial^{(\jmath)}\left(b_{(n+\jmath)} a\right)$,

(C4) $a_{(m)}\left(b_{(n)} c\right)-b_{(n)}\left(a_{(m)} c\right)=\sum_{\jmath=0}^{m}\left(\begin{array}{c}m \\ \jmath\end{array}\right)\left(a_{(\jmath)} b\right)_{(m+n-j)} c$.

Note that either of formulas (C2) follows from the other one and (C3).

Definition 2.3. A (Lie) conformal algebra is a $\mathbb{C}[\partial]$-module $R$, endowed with a family of $\mathbb{C}$-bilinear products ${ }_{(n)}, n \in \mathbb{Z}_{+}$, satisfying axioms (C1)-(C4). A conformal algebra $R$ is called finite if $R$ is a finitely generated $\mathbb{C}[\partial]$-module. The rank of a conformal algebra $R$, denoted by $\operatorname{rk} R$, is its rank as a $\mathbb{C}[\partial]$-module (recall that this is the dimension over $\mathcal{K}$, the field of fractions of $\mathbb{C}[\partial]$, of $\mathcal{K} \otimes_{\mathbb{C}[\partial]} R$ ).

Example 2.1 (Virasoro algebra and Virasoro conformal algebra). The centerless Virasoro algebra $\mathfrak{V e c t} \mathbb{C}^{\times}$is the Lie algebra of algebraic vector fields on $\mathbb{C}^{\times}$. It is spanned by vector fields $t^{n} \partial_{t}, n \in \mathbb{Z}$. The $\mathfrak{V e c t} \mathbb{C}^{\times}$-valued formal distribution $L(z)=-\sum_{n=-\infty}^{\infty}\left(t^{n} \partial_{t}\right) z^{-n-1}$ satisfies

$$
[L(z), L(w)]=\partial_{w} L(w) \delta(z-w)+2 L(w) \delta_{w}^{\prime}(z-w)
$$

and is therefore local to itself. The $\mathbb{C}[\partial]$-module generated by $L(z)$ is closed under all the products $(n)$ and thus forms a conformal family. The corresponding conformal algebra Vir is the free $\mathbb{C}[\partial]$-module on the generator $L$ with products

$$
L_{(0)} L=\partial L, L_{(1)} L=2 L, L_{(\jmath)} L=0 \text { for } j>1 .
$$

They uniquely extend to a conformal algebra structure by (C2).

Example 2.2 (Current algebras and current conformal algebras). The centerless current algebra associated to the Lie algebra $\mathfrak{g}$ is the space $\mathfrak{g}\left[t, t^{-1}\right]=\mathfrak{g} \otimes$ $\mathbb{C}\left[t, t^{-1}\right]$ endowed with the Lie bracket

$$
\left[g t^{m}, h t^{n}\right]=[g, h] t^{m+n}, g, h \in \mathfrak{g}, m, n \in \mathbb{Z}
$$

The $\mathfrak{g}\left[t, t^{-1}\right]$-valued formal distributions $g(z)=\sum_{n=-\infty}^{\infty} g t^{n} z^{-n-1}$ defined for every 
$g \in \mathfrak{g}$ satisfy the following commutation relations:

$$
[g(z), h(w)]=[g, h](w) \delta(z-w)
$$

and are therefore local to each other. Their $\mathbb{C}[\partial]$-linear span is closed under all products, and is therefore a conformal family. The conformal algebra describing this structure is given by the $\mathbb{C}[\partial]$-module $\operatorname{Cur} \mathfrak{g}=\mathbb{C}[\partial] \otimes \mathfrak{g}$ with the products

$$
g_{(0)} h=[g, h], g_{(i)} h=0 \text { for } i>0, g, h \in \mathfrak{g}
$$

where we identified $\mathfrak{g}$ with the subspace of $C u r \mathfrak{g}$ spanned by elements $1 \otimes g, g \in \mathfrak{g}$ as we will often do. The above products extend by $(\mathrm{C} 2)$ to a unique conformal algebra structure. The conformal algebra $C u r \mathfrak{g}$ is called the current conformal algebra associated to $\mathfrak{g}$.

Remark 2.2. When $R$ is a free $\mathbb{C}[\partial]$-module, and the products $(n)$ are defined on a $\mathbb{C}[\partial]$-basis for $R$ in such a way that $(\mathrm{C} 1),(\mathrm{C} 3)$ and (C4) hold, there is a unique extension of these products to a conformal algebra structure for $R$, obtained via $(\mathrm{C} 2)$, and it is easy to show that (C1) and (C4) also hold for this extension. We shall often describe conformal algebras structures on free $\mathbb{C}[\partial]$-modules by giving the products on a fixed $\mathbb{C}[\partial]$-basis.

The notions of subalgebras, ideals, quotients and homomorphisms of conformal algebras are obvious. Note that, due to (C3), any left or right ideal is actually a two-sided ideal.

As we have seen, any formal distribution Lie algebra $(\mathfrak{g}, \mathcal{F})$ gives rise to a conformal algebra $R=\overline{\mathcal{F}}$. Conversely, to any given conformal algebra $R$ we may canonically associate a formal distribution Lie algebra $\mathfrak{L} \mathfrak{i} R$ with a conformal family isomorphic to $R$ as follows $([\mathrm{K}],[\mathrm{K} 3])$. Let

$$
\mathfrak{L} \mathfrak{i} e R=R\left[t, t^{-1}\right] /\left(\partial+\partial_{t}\right) R\left[t, t^{-1}\right]
$$


let $a_{n}$ denote the image of $a t^{n}$ in $\mathfrak{L i e} R$, and define a bracket by

$$
\left[a_{m}, b_{n}\right]=\sum_{\jmath \in \mathbb{Z}_{+}}\left(\begin{array}{c}
m \\
j
\end{array}\right)\left(a_{(\jmath)} b\right)_{m+n-j}
$$

for $a, b \in R, m, n \in \mathbb{Z}$. This bracket is a well-defined Lie bracket and the family $\left\{a(z)=\sum_{n \in \mathbb{Z}_{+}} a_{n} z^{-n-1} \mid a \in R\right\}$ spans $\mathfrak{L i e} R$ and is a conformal algebra isomorphic to $R$ via $a(z) \mapsto a$. Furthermore, since $\partial$ and $\partial_{t}$ commute, the derivation $\partial_{t}$ of $R\left[t, t^{-1}\right]$ induces a derivation of $\mathfrak{L i e} R$.

Definition 2.4. The pair $(\mathfrak{L i e} R, R)$ is called the maximal formal distribution algebra associated to the conformal algebra $R$.

Remark 2.3. [K], [K3] The correspondence which associates to a conformal algebra $R$ the collection of quotients of $(\mathfrak{L i e} R, R)$ by ideals with a trivial intersection with $R$ is bijective. In particular, the axioms of a conformal algebra $R$ encode all the algebraic properties of the conformal family for $\mathfrak{L} \mathfrak{i} e R$. Axiom (C3) is equivalent to skew-simmetry and axiom (C4) to the Jacobi identity for the Lie algebra $\mathfrak{L i e} R$.

Remark 2.4. Since we know that the Jacobi identity on any triple of elements from a Lie algebra, together with skew-simmetry, give the Jacobi identity on all permutations of the triple, once (C1)-(C3) are established, it is enough to check (C4) on a triple of elements from a conformal algebra, in order for it to hold on all permutations of that triple. Therefore, if $R$ is a free $\mathbb{C}[\partial]$-module on generators $\left\{a^{i}, i=1 \ldots n\right\}$ and products $a_{(n)}^{2} a^{j}$ are defined for all $i \leq j$ and $n \in \mathbb{Z}_{+}$in such a way that (C3) holds for $a=b=a^{i}$ and (C4) holds for $a=a^{i}, b=a^{j}, c=a^{k}$ with $i \leq j \leq k$, we can extend them by $(\mathrm{C} 2)$ and (C3) to a unique conformal algebra structure for $R$. 


\section{Chapter 3}

\section{Conformal linear algebra}

From now on, $\mathcal{A}$ will denote the ring $\mathbb{C}[\partial]$ of polynomials in the indeterminate $\partial$.

Definition 3.1. A conformal linear map between $\mathcal{A}$-modules $V$ and $W$ is a map $\phi: V \rightarrow \mathcal{A}[\lambda] \otimes_{\mathcal{A}} W$ that is $\mathbb{C}$-linear and such that

$$
\phi \partial v=(\partial+\lambda)(\phi v) .
$$

We will often abuse the notation by writing $\phi: V \rightarrow W$ any time it is clear from the context that $\phi$ is conformal linear. We will also write $\phi_{\lambda}$ instead of $\phi$ to emphasize the dependence of $\phi$ on $\lambda$.

The set of all conformal linear maps from $V$ to $W$ is denoted by $\operatorname{Chom}(V, W)$ and is made into an $\mathcal{A}$-module via

$$
(\partial \phi)_{\lambda} v=-\lambda \phi_{\lambda} v .
$$

We shall write Cend $V$ for $\operatorname{Chom}(V, V)$.

Example 3.1. Let $F$ be a vector space and let $V=\mathcal{A} \otimes F$ be the corresponding free module over $\mathcal{A}$. A conformal linear map $\phi: V \rightarrow V$ is uniquely determined by its values on $1 \otimes F$. In fact

$$
\phi_{\lambda}(p(\partial) v)=p(\partial+\lambda)\left(\phi_{\lambda} v\right), v \in F
$$


determines the value of $\phi_{\lambda}$ on any element of $V$ from its value on $1 \otimes F$, and every End $F$-valued polynomial $a(\partial, \lambda)$ gives rise to such a conformal linear map via:

$$
\phi_{\lambda} v=a(\partial, \lambda) v
$$

We can therefore identify $\operatorname{Chom}(V, V)$ with the space of all End $F$-valued polynomials in $\partial$ and $\lambda$. Let $\left\{m_{\jmath}\right\}$ be a basis of End $F$; then the polynomials $m_{j}, m_{\jmath} \partial, m_{\jmath} \partial^{2}, \ldots$ are easily shown to be an $\mathcal{A}$-basis of $(\operatorname{End} F)[\partial, \lambda]$.

Hence $\operatorname{Chom}(V, V)$ is an infinite rank $\mathcal{A}$-module an $\mathcal{A}$-basis of which is given by conformal linear maps $T_{\jmath}^{0}, T_{\jmath}^{1}, T_{\jmath}^{2}, \ldots$ such that:

$$
\left(T_{j}^{\imath}\right)_{\lambda} v=\partial^{i}\left(m_{j} v\right), v \in F
$$

Let $a(z), u(w)$ be a local pair of formal distributions; we set

$$
a_{\lambda} u=\sum_{n \in \mathbb{Z}_{+}} \lambda^{(n)} a_{(n)} u
$$

Then we get by (C2):

$$
(\partial a)_{\lambda} u=-\lambda a_{\lambda} u, a_{\lambda} \partial u=(\partial+\lambda)\left(a_{\lambda} u\right)
$$

Example 3.2. Let $A$ be an associative algebra, $V$ an $A$-module and $\alpha: A \otimes V \rightarrow V$ the action of $A$ on $V$. If $\mathcal{V}$ is an $\mathcal{A}$-module of formal distributions with values in $V$, and $T(z)$ is an $A$-valued formal distribution local to all distributions from $\mathcal{V}$ with respect to the action induced by $\alpha$, then $T_{\lambda} v=\sum_{n \in \mathbb{Z}_{+}} \lambda^{(n)} T_{(n)} v$ defines a conformal linear map from $\mathcal{V}$ to itself. Furthermore, if $\mathcal{T}$ is the set of all $A$-valued formal distributions that are local to all members of $\mathcal{V}$, then the map $\pi: \mathcal{T} \rightarrow$ Cend $\mathcal{V}$, mapping $T$ to the conformal linear map it defines, commutes with the action of $\partial$ on both spaces, i.e. it is $\mathcal{A}$-linear. 
Let us now define, for $a, b$ in a conformal algebra $R$, the $\lambda$-bracket

$$
\left[a_{\lambda} b\right]=\sum_{n \in \mathbb{Z}_{+}} \lambda^{(n)} a_{(n)} b
$$

Then axioms $(\mathrm{C} 1)-(\mathrm{C} 4)$ are equivalently rephrased as follows:

(C1) $\left[a_{\lambda} b\right] \in \mathcal{A}[\lambda] \otimes_{\mathcal{A}} R$,

(C2) $\left[\partial a_{\lambda} b\right]=-\lambda\left[a_{\lambda} b\right], \quad\left[a_{\lambda} \partial b\right]=(\partial+\lambda)\left[a_{\lambda} b\right]$,

(C3) $\left[a_{\lambda} b\right]=-\left[b_{-\partial-\lambda} a\right]$,

(C4) $\left[a_{\lambda}\left[b_{\mu} c\right]\right]-\left[b_{\mu}\left[a_{\lambda} c\right]\right]=\left[\left[a_{\lambda} b\right]_{\lambda+\mu} c\right]$,

for all $a, b, c \in R$. The right-hand side of axiom (C3) (resp. (C4)) means an expression similar to (3.4), i.e. we replace $\lambda$ by $-\partial-\lambda$ (resp. by $\lambda+\mu$ ). Axiom (C4) is an equality in $\mathcal{A}[\lambda, \mu] \otimes_{\mathcal{A}} R$.

Example 3.3. Every $a \in R$ defines a conformal linear map ad $a: R \rightarrow R$ by

$$
(\operatorname{ad} a)_{\lambda} b=\left[a_{\lambda} b\right], b \in R
$$

In fact (C2) and (C3) imply:

$$
\left[a_{\lambda}(\partial b)\right]=-\left[(\partial b)_{-\partial-\lambda} a\right]=(-\partial-\lambda)\left[b_{-\partial-\lambda} a\right]=(\partial+\lambda)\left[a_{\lambda} b\right]
$$

Definition 3.2. Let $R$ be a conformal algebra. Then a conformal linear map $d: R \rightarrow R$ is a conformal derivation of $R$ if

$$
d_{\lambda}\left[a_{\mu} b\right]=\left[\left(d_{\lambda} a\right)_{\lambda+\mu} b\right]+\left[a_{\mu}\left(d_{\lambda} b\right)\right] .
$$

By (C4) ad $a$ is a conformal derivation of $R$ for every $a \in R$. All derivations of this kind are called inner.

A remarkable instance of a non-inner conformal derivation is the following.

Example 3.4 (Semi-direct sum of Vir and Cur $\mathfrak{g}$ ). Let $C u r \mathfrak{g}$ be the current conformal algebra associated to the finite-dimensional Lie algebra $\mathfrak{g}$. Define a conformal 
linear map $d^{L}:$ Cur $\mathfrak{g} \rightarrow$ Cur $\mathfrak{g}$ by $d_{\lambda}^{L} g=(\partial+\lambda) g$ for every $g \in \mathfrak{g} \subset$ Cur $\mathfrak{g}$. This is a conformal derivation since

$$
\begin{aligned}
& {\left[\left(d_{\lambda}^{L} g\right)_{\lambda+\mu} h\right]+\left[g_{\lambda}\left(d_{\mu}^{L} h\right)\right]=-\mu[g, h]+\left[g_{\lambda}(\partial+\mu) h\right]=} \\
& -\mu[g, h]+(\partial+\lambda+\mu)[g, h]=(\partial+\lambda)[g, h]=d_{\lambda}^{L}\left[g_{\mu} h\right]
\end{aligned}
$$

This derivation satisfies

$$
\left[d_{\lambda}^{L} d^{L}\right]=(\partial+2 \lambda) d^{L}
$$

and allows us to define a semi-direct sum of Vir and $C u r \mathfrak{g}$, called the standard semidirect sum. The $\mathcal{A}$-module $\operatorname{Vir} \oplus C u r \mathfrak{g}$ can in fact be given a conformal algebra structure by

$$
\left[L_{\lambda} L\right]=(\partial+2 \lambda) L, \quad\left[g_{\lambda} h\right]=[g, h], \quad\left[L_{\lambda} g\right]=d_{\lambda}^{L} g
$$

$L$ being the standard generator of $V i r, g, h \in \mathfrak{g}$. This $\lambda$-bracket is translated in terms of the $(n)$ products as follows:

$$
\begin{aligned}
& L_{(0)} L=\partial L, \quad L_{(1)} L=2 L, \quad L_{(0)} g=\partial g, \\
& L_{(1)} g=g, \quad g_{(1)} L=g, \quad g_{(0)} h=[g, h]
\end{aligned}
$$

where only the non-zero products of $L$ and elements from $\mathfrak{g} \subset C u r \mathfrak{g}$ have been given.

Example 3.5. The $\lambda$-bracket on $C e n d V$ given by

$$
\left[\phi_{\lambda} \psi\right]_{\mu} v=\phi_{\lambda}\left(\psi_{\mu-\lambda} v\right)-\psi_{\mu-\lambda}\left(\phi_{\lambda} v\right)
$$

defines a conformal algebra structure on Cend $V$ since it satisfies properties (C1)(C4). This is called the general conformal algebra on $V$ and is denoted by $g c V$. The set $\operatorname{Der} R$ of all conformal derivations of a conformal algebra $R$ is a subalgebra of $g c R$. 
If $V=\mathcal{A}^{N}$ is a free $\mathcal{A}$-module of rank $N$, then $g c V$ is also denoted by $g c_{N}$. We have already seen that the action of a conformal map on $V$ is determined by its action on an $\mathcal{A}$-basis of $V$ via the compatibility relation

$$
\phi_{\lambda}(p(\partial) v)=p(\partial+\lambda) \phi_{\lambda} v
$$

so that the conformal linear maps $T_{A}^{m}$ acting as $\left(T_{A}^{m}\right)_{\lambda} v=\partial^{m} A v$, $A \in \operatorname{End} V, v \in V$, have the following $\lambda$-brackets:

$$
\left[T_{A \lambda}^{m} T_{B}^{n}\right]=\sum_{i=0}^{\infty}\left(\begin{array}{c}
n \\
\imath
\end{array}\right) \lambda^{\imath} T_{A B}^{m+n-\imath}-\left(\begin{array}{c}
m \\
i
\end{array}\right)(-\partial-\lambda)^{i} T_{B A}^{m+n-i}
$$

as one can easily check by applying both sides to any $v \in V$ and using Equation (3.6).

Let $V$ be an $\mathcal{A}$-module, $v(\lambda)=\sum_{\imath=0}^{n} \lambda^{2} v_{\imath} \in \mathcal{A}[\lambda] \otimes_{\mathcal{A}} V$, where $v_{\imath} \in V$. Define $\langle v\rangle$ to be the $\mathcal{A}$-submodule of $V$ spanned by all coefficients $v_{\imath}$ of $v$. The following lemma will be very useful.

Lemma 3.1. If $p(\lambda)=\sum_{\imath=0}^{m} p_{\imath} \lambda^{2} \in \mathcal{A}[\lambda]$ is a polynomial whose leading coefficient does not depend on $\partial$ (i.e. is a complex number different from zero), then $\langle p v\rangle=\langle v\rangle$.

Proof. We show, by induction on $h$, that all $v_{n-h}$ lie in $\langle p v\rangle$. Let $p(\lambda) v(\lambda)=$ $\sum_{j=0}^{m+n} \lambda^{\jmath}(p v)_{\jmath}$. Then $(p v)_{m+n}=p_{m} v_{n}$ and hence $v_{n} \in\langle p v\rangle$. Suppose now $v_{n_{-i}} \in$ $\langle p v\rangle$ for all $i \leq h$; we want to show that $v_{n-h-1}$ also lies in $\langle p v\rangle$. We have that $(p v)_{m+n-h-1}=p_{m} v_{n-h-1}+p_{m-1} v_{n-h}+\ldots+p_{m-h-1} v_{n} \in\langle p v\rangle$. But by inductive hypothesis, all terms but the first one in the right hand side already lie there. Hence $p_{m} v_{n-h-1}$, and $v_{n-h-1}$, also lie in $\langle p v\rangle$. The other inclusion $\langle p v\rangle \subset\langle v\rangle$ is trivial.

The torsion of a conformal algebra $R$, denoted Tor $R$ is the torsion of the $\mathcal{A}$ module $R$, namely the submodule of all elements $r$ for which there exists a non-zero $p \in \mathcal{A}$ such that $p(\partial) r=0$. The following proposition shows that the torsion does not play any significant role in the conformal algebra structure.

Proposition 3.1. The torsion Tor $R$ always lies in the center $Z(R)=\left\{r \in R \mid\left[r_{\lambda} s\right]=0\right.$ for all $\left.s \in R\right\}$ of $R$. 
Proof. Let $t \in$ Tor $R$. Then there is a non-zero $p \in \mathcal{A}$ killing $t$ and we have:

$$
0=\left[0_{\lambda} r\right]=\left[p(\partial) t_{\lambda} r\right]=p(-\lambda)\left[t_{\lambda} r\right]
$$

for all $r \in R$. This proves $\left\langle p(-\lambda)\left[t_{\lambda} r\right]\right\rangle=0$, hence by Lemma 3.1 $\left\langle\left[t_{\lambda} r\right]\right\rangle=0$, which shows $\left[t_{\lambda} r\right]=0$ for every $r \in R$.

Remark 3.1. The above proof actually shows that if $\phi$ is a conformal linear map such that $p(\partial) \phi=0$ for a non-zero $p \in \mathcal{A}$, then $\phi=0$.

In view of Proposition 3.1 the $\lambda$-bracket of a conformal algebra $R$ defines an adjoint map ad $: R \rightarrow \operatorname{Der} R \subset g c R$ whose kernel is the center of $R$ and which is a homomorphism of conformal algebras. Thus all finite rank centerless conformal algebras can be embedded as finite rank conformal subalgebras of $g c_{N}$.

Proposition 3.2. A conformal linear map $\phi: V \rightarrow W$ always maps Tor $V$ to zero.

Proof. Say $v \in V$ is torsion. Then there is a non-zero $p \in \mathcal{A}$ such that $p(\partial) v=0$. Then $0=\phi_{\lambda}(p(\partial) v)=p(\partial+\lambda)\left(\phi_{\lambda} v\right)$. Use now Lemma 3.1 to conclude that $\phi_{\lambda} v=$ 0 .

We now classify all conformal algebras that are free of rank one as $\mathcal{A}$-modules. This is a joint result with M.Wakimoto.

Proposition 3.3. Let $R=\mathcal{A} x$ be a conformal algebra that is free of rank one as an $\mathcal{A}$-module. Then either $R$ is commutative, i.e. the $\lambda$-bracket is 0 , or it is isomorphic to Vir.

Proof. Axioms (C4) and (C3) give the following relations:

$$
\begin{gathered}
{\left[x_{\lambda}\left[x_{\mu} x\right]\right]-\left[x_{\mu}\left[x_{\lambda} x\right]\right]=\left[\left[x_{\lambda} x\right]_{\lambda+\mu} x\right]} \\
{\left[x_{\lambda} x\right]=-\left[x_{-\partial-\lambda} x\right] .}
\end{gathered}
$$


Let us set $\left[x_{\lambda} x\right]=a(\partial, \lambda) x$ for some polynomial $a$. Then (3.8) is equivalent to

$$
a(\partial, \lambda) a(\partial+\lambda, \mu)-a(\partial, \mu) a(\partial+\mu, \lambda)=a(\partial, \lambda+\mu) a(-\lambda-\mu, \lambda) .
$$

Let $a(\partial, \lambda)=\sum_{i=0}^{n} a_{i}(\lambda) \partial^{2}$ with $a_{n} \neq 0$. Then, asșuming $n>1$, if we equate terms of degree $2 n-1$ in $\partial$, we get

$$
n(\lambda-\mu) a_{n}(\lambda) a_{n}(\mu)=0
$$

obtaining a contradiction. So $a(\partial, \lambda)=\alpha(\lambda) \partial+\beta(\lambda)$ is linear in $\partial$. If we put $\lambda=\mu$ in (3.10), we get $a(\partial, 2 \lambda) a(-2 \lambda, \lambda)=0$, which means $\beta(\lambda)=2 \lambda \alpha(\lambda)$, therefore $a(\partial, \lambda)=\alpha(\lambda)(\partial+2 \lambda)$. Plugging this into (3.9), we get that $\alpha$ is a constant. Up to changing $x$ by a complex multiple we can make it 0 or 1 . In the first case we have the trivial product, in the second the Virasoro algebra.

The analogy between ordinary linear algebra and conformal linear algebra is very suggestive of the importance of other remarkable constructions. We have already seen that the family of all conformal maps between two $\mathcal{A}$-modules can itself be given an $\mathcal{A}$-module structure.

The notion of associative algebra (and of Lie algebra) are modelled on the algebra of linear maps between vector spaces. It is natural to try to extend this construction to the conformal case.

Say we have conformal linear maps $\phi: V \rightarrow W$ and $\psi: U \rightarrow V$ where $U, V, W$ are $\mathcal{A}$-modules. We would like to define the notion of the composition of $\phi$ and $\psi$ resulting in a new conformal linear map from $U$ to $W$. Conformal linearity implies $\phi_{\lambda} \partial v=(\partial+$ $\lambda)\left(\phi_{\lambda} v\right)$,

$\psi_{\mu} \partial u=(\partial+\mu)\left(\psi_{\mu} u\right)$, so that the obvious composition would satisfy $\phi_{\lambda}\left(\psi_{\mu} \partial u\right)=$ $(\partial+\lambda+\mu)\left(\phi_{\lambda}\left(\psi_{\mu} u\right)\right)$. Our composition must therefore be a conformal map indexed by $\lambda+\mu$. The natural choice is:

$$
\left(\phi_{\lambda} \psi\right)_{\lambda+\mu} u=\phi_{\lambda}\left(\psi_{\mu} u\right)
$$


for all $u \in U$. This is the only possible choice to be compatible with replacing $\phi$ by $\partial \phi$ or $\psi$ by $\partial \psi$. This composition satisfies (3.3), and defines on $C e n d V$ a structure of an associative conformal algebra as defined below:

Definition 3.3. An $\mathcal{A}$-module $A$ is an associative conformal algebra if it is endowed with a $\lambda$-product $A \otimes A \ni a \otimes b \mapsto a_{\lambda} b \in \mathcal{A}[\lambda] \otimes_{\mathcal{A}} A$ such that for all $a, b, c \in A$ the following two axioms hold:

$$
\begin{aligned}
& \text { (A1) }(\partial a)_{\lambda} b=-\dot{\lambda} a_{\lambda} b, \quad a_{\lambda} \partial b=(\partial+\lambda) a_{\lambda} b \\
& \text { (A2) } a_{\lambda}\left(b_{\mu} c\right)=\left(a_{\lambda} b\right)_{\lambda+\mu} c
\end{aligned}
$$

The next step is taking the commutator in $A$ in order to define the analogue of the notion of a Lie algebra. The properties we want to retain of the commutator in an associative algebra are the bilinearity in the arguments, that we know translates as (C2), and skew-simmetry (C3). There is a unique reasonable choice:

$$
\left[x_{\lambda} y\right]=x_{\lambda} y-y_{-\partial-\lambda} x
$$

It follows from Remark 3.2 below (and it is not difficult to show directly using Remark 3.3) that this $\lambda$-bracket defines a (Lie) conformal algebra structure on $A$. It is also easy to see that the $\lambda$-bracket defined by (3.6) coincides with the one defined by $(3.12)$.

Remark 3.2. A formal distribution associative algebra $(A, \mathcal{F})$ gives rise to a conformal associative algebra $\overline{\mathcal{F}}$ in the same way as in the Lie case. Similarly one constructs the maximal formal distribution associative algebra $(\operatorname{Alg} R, R)$ attached to an associative conformal algebra $R$. The correspondence between $R$ and $\operatorname{Alg} R$ is the same as described by Remark 2.3 in the Lie case. Furthermore, passing from a formal distribution associative algebra $(A, \mathcal{F})$ to the Lie algebra $(A, \mathcal{F})$ (obtained from $A$ by imposing the bracket $[a, b]=a b-b a$ ) corresponds to passing from the $\lambda$-product $a_{\lambda} b$ on $\mathcal{F}$ to the $\lambda$-bracket (3.12).

Remark 3.3. The following properties always hold in an associative conformal alge- 
bra:

$$
\begin{gathered}
a_{\lambda}\left(b_{-\partial-\mu} c\right)=\left(a_{\lambda} b\right)_{-\partial-\mu} c \\
a_{-\partial-\lambda}\left(b_{\mu} c\right)=\left(a_{-\partial-\mu} b\right)_{-\partial+\mu-\lambda} c \\
a_{-\partial-\lambda}\left(b_{-\partial-\mu} c\right)=\left(a_{-\partial+\mu-\lambda} b\right)_{-\partial-\mu} c .
\end{gathered}
$$




\section{Chapter 4}

\section{The annihilation algebra}

We have seen in Chapter 2 how one can associate to a formal distribution Lie algebra a conformal algebra. We have also inverted this construction, i.e. to a given conformal algebra $R$ we have associated its maximal formal distribution Lie algebra $(\mathfrak{L i e} R, R)$.

Let now $R$ be a finite conformal algebra. Then we may find a free complementary $\mathcal{A}$-submodule $R_{f}$ to $\operatorname{Tor} R$ :

$$
R=R_{f} \oplus \text { Tor } R,
$$

where $R_{f}=\mathcal{A} \otimes \mathfrak{g}$, and $\mathfrak{g} \subset R$ is a finite-dimensional subspace. Then we have the identification $[\mathrm{K}]$

$$
\mathfrak{L} \mathfrak{i} R=\mathfrak{g}\left[t, t^{-1}\right] \oplus(\text { Tor } R) t^{-1}
$$

with the following brackets (cf. (2.11)):

$$
\begin{gathered}
{\left[a_{m}, b_{n}\right]=\sum_{j \in \mathbb{Z}_{+}}\left(\begin{array}{c}
m \\
j
\end{array}\right)\left(a_{(j)} b\right)_{m+n-j},} \\
{\left[\mathfrak{L i e} R,(\text { Tor } R) t^{-1}\right]=0}
\end{gathered}
$$


where $a, b \in \mathfrak{g}, m, n \in \mathbb{Z}_{+}, a_{n}$ stands for $a t^{n}$, and we use the rule

$$
(\partial a)_{m}=-m a_{m-1}, a \in R, m \in \mathbb{Z}
$$

Recall also that $\mathfrak{L} \mathfrak{i} \mathfrak{e}$ admits a derivation $\partial$ defined by

$$
\partial\left(a_{n}\right)=-n a_{n-1}, a \in R, n \in \mathbb{Z}
$$

Thus, $\mathfrak{L} \mathfrak{i e} R$ may be viewed as a generalization of a current Lie algebra. We have the following corollaries of the above remarks and Remark 2.3.

Proposition 4.1. Let $(\mathfrak{g}, \mathcal{F})$ be a formal distribution Lie algebra with a conformal family $\mathcal{F}$, which is a free $\mathcal{A}$-module on a basis $B$. Suppose that all elements $a_{n}, a \in$ $B, n \in \mathbb{Z}$, are linearly independent. Then $(\mathfrak{g}, \mathcal{F})$ is isomorphic to the maximal formal distribution algebra associated to the conformal algebra $\mathcal{F}$. A similar statement is true for formal distribution associative algebras.

Proposition 4.2. Let $R$ be a finite conformal algebra and let $\left\{a^{\alpha}\right\}$ be a finite set of generators for $R$ (as an $\mathcal{A}$-module). Let $\mathfrak{L}_{m}$ be the linear span of $\left\{a_{i}^{\alpha}, i \geq m\right\}$; then we have a "quasi-filtration" of $\mathfrak{L}_{0}$ :

$$
\mathfrak{L}_{0} \supset \mathfrak{L}_{1} \supset \mathfrak{L}_{2} \supset \ldots
$$

by subspaces $\mathfrak{L}_{\imath}$ of finite codimension satisfying

$$
\left[\mathfrak{L}_{i}, \mathfrak{L}_{j}\right] \subset \mathfrak{L}_{i+j-s} \text { for some } s \in \mathbb{Z}_{+} \text {, all } i, j \in \mathbb{Z} \text {, and }\left[\partial, \mathfrak{L}_{i}\right]=\mathfrak{L}_{i-1}
$$

$\left(\right.$ we let $\mathfrak{L}_{j}=\mathfrak{L}_{0}$ if $\left.j<0\right)$.

Proof. We have:

$$
\left[a_{\lambda}^{\alpha} a^{\beta}\right]=\sum_{\gamma} p_{\gamma}^{\alpha \beta}(\partial, \lambda) a^{\gamma}, \text { some } p_{\gamma}^{\alpha \beta}
$$

Let $s$ be the highest degree of the polynomials $p_{\gamma}^{\alpha \beta}$. The statement clearly follows from (2.11). 
Remark 4.1. One can use the family $\left\{\mathfrak{L}_{m}\right\}$ to define a topology on $(\mathfrak{L} \mathfrak{i} e R)_{-}$by setting $\left\{\mathfrak{L}_{m}\right\}$ to be a basis of neighbourhoods of zero. The quasi-filtration $\left\{\mathfrak{L}_{m}\right\}$ depends on the choice of the set of generators for $R$, but the topology it induces on $\mathfrak{L}_{0}$ is independent of that choice. The Lie bracket on $\mathfrak{L}_{0}$ and the action of $\partial$ are clearly continuous.

Definition 4.1. The annihilation algebra associated to a conformal algebra $R$ is the subalgebra

$$
(\mathfrak{L} \mathfrak{i} e R)_{-}=\mathfrak{L}_{0}=\left\{a_{n} \mid a \in R, n \geq 0\right\}
$$

of the Lie algebra $\mathfrak{L i e} R$, topologized as in Remark 4.1. Obviously, $(\mathfrak{L i e} R)_{-}$is $\partial$ invariant. The semi-direct sum $(\mathfrak{L i e} R)^{-}=\mathbb{C} \partial+(\mathfrak{L i e} R)_{-}$is called the extended annihilation algebra.

Equations (4.1)-(4.5) show that $(\mathfrak{L i e} R)_{-}$is nothing but $\mathfrak{g}[t]$ with the Lie bracket (4.2). We can describe now (extended) annihilation algebras in all important examples.

Example 4.1. Let us consider the Virasoro conformal algebra Vir, and let $L$ be its standard generator. Then by Proposition 4.1, $\mathfrak{L i e V i r}$ is isomorphic to $\mathfrak{V e c t} \mathbb{C}^{\times}$ under the identification $L_{j}=-t^{j} \partial_{t}$. The associated annihilation algebra is the Lie subalgebra spanned by $t^{3} \partial_{t}$ with $j \geq 0$. In other words, $(\mathfrak{L i e V i r})_{-} \simeq \mathfrak{V e c t} \mathbb{C}$. Its topology is the one induced from the standard filtration $\left\{\mathfrak{L}_{n}=t^{n} \mathbb{C}[t] \partial_{t}\right\}$ of $\mathfrak{V e c t} \mathbb{C}$. Since $\partial$ acts on it as $-\operatorname{ad} \partial_{t}$, we see that $(\mathfrak{L i e V i r})^{-}$is isomorphic to the direct sum of $\mathfrak{V e c t} \mathbb{C}$ and the one-dimensional Lie algebra $\mathbb{C}\left(\partial+\partial_{t}\right)$.

Example 4.2. The Lie algebra $\mathfrak{L i e} C u r \mathfrak{g}$, where $C u r \mathfrak{g}$ is the current conformal algebra associated to a complex Lie algebra $\mathfrak{g}$, is isomorphic to $\mathfrak{g}\left[t, t^{-1}\right]=\mathfrak{g} \otimes_{\mathbb{C}} \mathbb{C}\left[t, t^{-1}\right]$ where we identified $g_{n}$ with $g t^{n}$. The corresponding annihilation algebra is its positive part $\mathfrak{g}[t]$, its topology being the one induced by the standard filtration $\left\{\mathfrak{L}_{n}=t^{n} \mathfrak{g}[t]\right\}$, and $\partial$ acts on this as $-\partial_{t}$.

Example 4.3. Let Diff $\mathbb{C}^{\times}$be the associative algebra of algebraic differential operators on $\mathbb{C}^{\times}$. Its elements are $\sum_{\imath=0}^{k} p_{\imath}(t) \partial_{t}^{i}$ where $p_{\imath} \in \mathbb{C}\left[t, t^{-1}\right]$. A basis for Diff $\mathbb{C}^{\times}$is 
given by $\left\{t^{m} \partial_{t}^{n} \mid m \in \mathbb{Z}, n \in \mathbb{Z}_{+}\right\}$, and the product is given by the usual composition of operators. Denote by $\left(\text { Diff } \mathbb{C}^{\times}\right)_{N}$ the algebra End $\mathbb{C}^{N} \otimes$ Diff $\mathbb{C}^{\times}$of End $\mathbb{C}^{N}$-valued differential operators on $\mathbb{C}^{\times}$. It acts naturaily on the vector space $\mathbb{C}^{N} \otimes \mathbb{C}\left[t, t^{-1}\right]$. The $\left(\text { Diff } \mathbb{C}^{\times}\right)_{N}$-valued formal distributions

$$
J_{A}^{k}(z)=\sum_{n \in \mathbb{Z}} A t^{n}\left(-\partial_{t}\right)^{k} z^{-n-1}
$$

act on the set $v(z)=\sum_{n}\left(v t^{n}\right) z^{-n-1}=v \delta(z-t), v \in \mathbb{C}^{N}$, of $\mathbb{C}^{N}$-valued formal distributions. Since $J_{A}^{k}(z)=A \delta(z-t)\left(-\partial_{t}\right)^{k}, J_{A}^{k}$ and $v$ are local under composition, explicitly:

$$
\begin{aligned}
& J_{A}^{k}(z) v(w)=A \delta(z-t)\left(-\partial_{t}\right)^{k} v \delta(w-t)= \\
& =A v \partial_{w}^{k}(\delta(z-t) \delta(w-t))=A v \sum_{i=0}^{k}\left(\begin{array}{c}
k \\
i
\end{array}\right) \partial_{w}^{k-\imath} \delta(w-t) \partial_{w}^{2} \delta(z-w) \\
& =\sum_{i=0}^{k}\left(\begin{array}{c}
k \\
i
\end{array}\right) \partial^{k-\imath} A v(w) \partial_{w}^{2} \delta(z-w) .
\end{aligned}
$$

Hence $J_{A}^{k}$ induces a conformal linear map of $\mathcal{A} \otimes \mathbb{C}^{N}$ to itself, given in terms of $\lambda$-product as

$$
\left(J_{A}^{k}\right)_{\lambda} v=\sum_{i=0}^{k}\left(\begin{array}{c}
k \\
i
\end{array}\right) \lambda^{\imath} \partial^{k-\imath} A v=(\partial+\lambda)^{k} A v .
$$

Since the $J_{A}^{k}$ 's act locally on an $\mathcal{A}$-module, they are local to each other with respect to the $\lambda$-product on Cend $\mathcal{A}^{N}$. If $\left\{m_{i}\right\}$ is a basis of End $\mathbb{C}^{N}$, then the formal distributions $J_{m_{2}}^{k}$ form a basis of $\operatorname{Cend} \mathcal{A}^{N}$ over $\mathcal{A}$ (this is the same as to say that polynomials $(\partial+\lambda)^{k}$ form an $\mathcal{A}$-basis for $\left.\mathbb{C}[\partial, \lambda]\right)$ and Fourier coefficients of all the $J_{m_{2}}^{k}$ are linearly independent, hence by Proposition 4.1 the associative algebra $\operatorname{Alg} C e n d \mathcal{A}^{N}$ (see Remark 3.2) is isomorphic to (Diff $\left.\mathbb{C}^{\times}\right)_{N}$. Simplicity of $\left(\text { Diff } \mathbb{C}^{\times}\right)_{N}$ then implies simplicity of Cend $\mathcal{A}^{N}$ as an associative conformal algebra.

The same is true for $g c_{N}$ : the Lie algebra $\mathfrak{L} \mathfrak{i} g c_{N}$ is isomorphic to the Lie algebra $\left(\mathfrak{D i f f} \mathbb{C}^{\times}\right)_{N}$ of End $\mathbb{C}^{N}$-valued algebraic differential operators on $\mathbb{C}^{\times}$. The annihilation algebra of Cend $\mathcal{A}^{N}$ (resp. $g c_{N}$ ) is obviously isomorphic to the polynomial part of $\left(\text { Diff } \mathbb{C}^{\times}\right)_{N}$ with the usual product (resp. Lie bracket). 
Remark 4.2. The $J_{A}^{k}$ 's constitute an alternative basis for $g c_{N}$ to the one considered in Example 3.1.

Now we discuss some properties of annihilation algebras that will turn out useful in the sequel.

Introduce the following generating series of elements of $(\mathfrak{L} \mathfrak{i} R)_{-}: a_{\lambda}=\sum_{n} \lambda^{(n)} a_{n}$. Then we have an equivalent form of (2.11) and (4.4):

$$
\begin{gathered}
{\left[a_{\lambda}, b_{\mu}\right]=\left[a_{\lambda} b\right]_{\lambda+\mu},} \\
(\partial a)_{\lambda}=-\lambda a_{\lambda} .
\end{gathered}
$$

Replacing $\mu$ by $\mu-\lambda$, we get $\left[a_{\lambda} b\right]_{\mu}=\left[a_{\lambda}, b_{\mu-\lambda}\right]$ or, equivalently

$$
\left(a_{(m)} b\right)_{n}=\sum_{\jmath=0}^{m}(-1)^{m+\jmath}\left(\begin{array}{c}
m \\
j
\end{array}\right)\left[a_{\jmath}, b_{m+n-\jmath}\right]
$$

Lemma 4.1. $a_{\lambda}=0$ if and only if $a \in$ Tor $R$.

Proof. By (4.1) and (4.8) if $a \notin$ Tor $R$, then $a_{n} \neq 0$ for $n$ sufficiently large, and if $a \in \operatorname{Tor} R$ then $a_{n}=0$ for all non-negative $n$.

Lemma 4.2. A torsionless conformal algebra $R$ is commutative if and only if its annihilation algebra $(\mathfrak{L} \mathfrak{i} R)_{-}$is commutative.

Proof. If the $\lambda$-bracket is $0,(\mathfrak{L i e} R)_{-}$is trivially commutative. Suppose now $(\mathfrak{L i e} R)_{-}$ is a commutative Lie algebra. From (4.7), we get $\left[a_{\lambda} b\right]_{\lambda+\mu}=0$ which shows $\left[a_{\lambda} b\right] \in$ Tor $R$ because of Lemma 4.1.

An ideal $\mathfrak{j} \subset(\mathfrak{L i e} R)_{-}$is said to be regular if there is some ideal $J \subset R$ whose Fourier coefficients span $\mathfrak{j}$; then $\mathfrak{j}$ is stable under the action of $\partial$.

Lemma 4.3. If $R$ is a torsionless conformal algebra, then every $\partial$-stable ideal $\mathfrak{J}$ of $(\mathfrak{L i e} R)_{\text {_ }}$ that is not contained in the center of $(\mathfrak{L} \mathfrak{i} e R)_{\text {_ contains a non-zero regular }}$ subideal. 
Proof. Suppose there is an $a \in R$ such that $a_{m} \in \mathfrak{J}$ for all $m \in \mathbb{Z}_{+}$. Then (4.7) shows that $\left(a_{(\imath)} b\right)_{j} \in \mathfrak{J}$ for all $b \in R, i, j \in \mathbb{Z}_{+}$. Hence the ideal of $(\mathfrak{L i e} R)_{-}$generated by $\left\{a_{m}, m \in \mathbb{Z}_{+}\right\}$, which is clearly contained in $\mathfrak{J}$, is $(\mathfrak{L} \mathfrak{i} J)_{\text {_ }}$ where $J$ is the ideal of $R$ generated by $a$.

Therefore, we are only left with proving that there is such an $a$. Choose an element $x_{n} \in \mathfrak{J}$ not lying in the center of $(\mathfrak{L i e} R)_{-}$. There exists a maximal $i$ such that all the $x_{j}, j<i$ lie in the center of $\mathfrak{J}$. It is clear by (4.9) that $x_{(\jmath)} y=0$ for all $j<i, y \in R$, so that by $(2.11)\left[x_{\imath}, y_{m}\right]=\left(x_{(\imath)} y\right)_{m}$, for all $m$. So $a=x_{(i)} y$ is the element we are looking for.

Remark 4.3. The proof of Lemma 4.3 actually shows that $\left[(\mathfrak{L i e} R)_{-},\left[(\mathfrak{L i e} R)_{-}, \mathfrak{J}\right]\right]$ is always a regular ideal. It also follows from (2.11) and (4.9), as well as the proof of Lemma 4.3 , that when $\mathfrak{a}$ and $\mathfrak{b}$ are regular ideals, the ideal $[\mathfrak{a}, \mathfrak{b}]$ is also regular. In particular, if $\mathfrak{a}$ (resp. $\mathfrak{b}$ ) is spanned by all Fourier coefficients of $A$ (resp. $B$ ) for ideals $A, B$ of a conformal algebra $R$, then $[\mathfrak{a}, \mathfrak{b}]$ is regular, and is induced by $\left\langle\left[A_{\lambda} B\right]\right\rangle$ (see Lemma 3.1).

Definition 4.2. The completed (resp. completed extended) annihilation algebra of a conformal algebra $R$, denoted $\left(\widehat{\mathfrak{L i e} R)_{-}}\left(\right.\right.$resp. $\left(\widehat{\mathfrak{L i e} R)^{-}}\right)$, is the completion of $(\mathfrak{L i e} R)_{-}$ (resp. $\left.(\mathfrak{L i e} R)^{-}\right)$with respect to its topology.

For example, the completed annihilation algebra for $C u r \mathfrak{g}$ is $\mathfrak{g}[[t]]$, while that for Vir is $\mathbb{C}[[t]] \partial_{t}$. In both cases the topology is induced by filtering with respect to the power series degree in $\mathrm{t}$.

There is a conformal module action of $(\mathfrak{L i e} R)_{\text {- on }} R$ given by $r_{n} . s=r_{(n)} s$, which we will call the adjoint conformal representation of $R$ (see Section 8). Since for any $r \in R, \mathfrak{L}_{k} . r=0$ for $k>>0$, this action extends to the whole $\left(\widehat{\mathfrak{L i}_{\mathfrak{i} R} R}\right)_{-}$.

Lemma 4.4. Let $x \in\left(\widehat{(\mathfrak{\mathfrak { i } e} R)_{-}}, r \in R\right.$. Then we have:

$$
\left[x, r_{n}\right]=\sum_{i=0}^{\infty}\left((-\partial)^{(i)} x \cdot r\right)_{n+i}
$$


and

$$
(x . r)_{n}=\sum_{i=0}^{\infty}\left[\partial^{(i)} x, r_{n+2}\right]
$$

Proof. It is enough to show (4.10) for $x=a_{m}, a \in R, m \in \mathbb{Z}_{+}$. Density of $(\mathfrak{L i e} R)_{-}$ along with convergence of both sides in (4.10) will give the general statement. But $\left[a_{m}, r_{n}\right]$ and $\sum_{i=0}^{\infty}\left((-\partial)^{(i)} a_{m} . r\right)_{n+i}$ both equal $\sum_{\jmath=0}^{\infty}\left(\begin{array}{c}m \\ j\end{array}\right)\left(a_{(\jmath)} r\right)_{m+n-j}$, since $a_{k} \cdot r=$ $a_{(k)} r$. The second equality then follows by substituting (4.10) in the right-hand side of (4.11).

Proposition 4.3. Let $R$ be a torsionless conformal algebra, and let $\mathfrak{j}$ be a $\partial$-stable ideal of $\left(\widehat{\mathfrak{L i e} R)_{-}}\right.$such that $\mathfrak{j} \cap\left(\mathfrak{L i e} R_{-}=0\right.$. Then $\mathfrak{j}$ is central in $\left(\widehat{\mathfrak{L i e} R)_{-}}\right.$.

Proof. Let $x \in \mathfrak{j}, r \in R$. Then because of $(4.11),(x . r)_{n}$ lies in $\mathfrak{j}$. But $x . r$ belongs to $R$, hence $(x . r)_{n}$ also lies in $(\mathfrak{L i e} R)_{-}$. This shows that $(x . r)_{n} \in \mathfrak{j} \cap(\mathfrak{L i e} R)_{-}=0$, and since $R$ is torsionless, x.r must equal 0. Thus $x . r=0$ for all $x \in \mathfrak{j}, r \in R$, which means, by Lemma 4.4 , that $x \in \mathrm{j}$ is always central in $(\widehat{\mathfrak{\mathcal { i e } R} R})_{-}$. 


\section{Chapter 5}

\section{Simple conformal algebras}

We now want to undertake the task of studying simple conformal algebras through the properties of the corresponding annihilation algebras; throughout this section, annihilation algebras will always be considered along with their topology.

Definition 5.1. A conformal algebra $R$ is simple if it is non-commutative and has no non-trivial ideals.

It is clear that Vir is simple, and that $C u r \mathfrak{g}$ is simple if and only if $\mathfrak{g}$ is a simple Lie algebra.

Remark 5.1. In consideration of Proposition 3.1, any simple conformal algebrä $R$ must be torsionless.

The final goal of this section is the proof of the following

Theorem 5.1. A finite simple conformal algebra is isomorphic either to Vir or to the current conformal algebra Cur $\mathfrak{g}$ associated to a simple finite-dimensional Lie algebra $\mathfrak{g}$.

The most important result we are going to exploit in the proof of this statement is a theorem by Cartan and Guillemin $[G]$ which classifies linearly compact topological Lie algebra satisfying certain additional properties. We will therefore need a few generalities (see e.g. [G]) about linearly compact topological spaces. 
Let $V$ be a vector space. We will say that a topology for $V$ is linear if there is a basis of open sets consisting only of affine subsets of $V$. We will call $V$ an affine topological vector space. An affine topological vector space $V$ is linearly compact if it satisfies the finite intersection property on families of closed affine subsets, i.e. if $\cap_{2} F_{i} \neq$ for every family $\left\{F_{2}\right\}_{2 \in I}$ of closed affine subsets of $V$ having non-empty finite intersections. For a topological vector space $V$ the property of being linearly compact is equivalent to being the topological product of spaces isomorphic to a onedimensional vector space with the discrete topology. Also, a topological vector space is linearly compact if and only if its topological dual is a discrete topological space. A linearly compact Lie algebra is a topological Lie algebra which is linearly compact as a topological vector space.

The Lie algebra $(\widehat{\mathfrak{L i e} R)}-$, topologized as in Definition 4.2 , is linearly compact as a topological vector space, since it is the product of $\mathbb{C} \partial$ and $\mathfrak{L}_{\imath} / \mathfrak{L}_{i+1}, i>0$.

Lemma 5.1. If $R$ is a finite conformal algebra, then $(\widehat{\mathfrak{\mathcal { L i e } R})}-$ has a closed subalgebra of finite codimension containing no ideals of $\left(\widehat{\mathfrak{L i e} R)^{-}}\right.$.

Proof. By (4.1) we can assume $R$ to be torsionless. Use Proposition 4.2 with an $\mathcal{A}$ basis of $R$. The closure of $\mathfrak{L}_{1}$ in $\left(\widehat{\mathfrak{L i k} R)^{-}}\right.$does not contain any ideal of $\left(\widehat{\mathfrak{L i k} R}^{-}\right.$, and is clearly of finite codimension.

Remark 5.2. A subspace of a linearly compact topological vector space is open if and only if it is closed and of finite codimension. So we have just shown that $\left(\widehat{\mathfrak{L i e} R)^{-}}\right.$ has an open subalgebra. This is important in view of next theorem (notice that the topological tensor product $V \widehat{\otimes} W$ of two linearly compact vector spaces is defined as the topological dual of $V^{*} \otimes W^{*}$ endowed with the discrete topology).

Theorem 5.2. [B] Let $\mathfrak{L}$ be a linearly compact Lie algebra having an open subalgebra containing no ideals of $\mathfrak{L}$. Then:

(a) Every non-commutative minimal closed ideal of $\mathfrak{L}$ is of the form $\mathfrak{S}=S \widehat{\otimes} \mathbb{C}\left[\left[t_{1}, t_{2}, \ldots, t_{r}\right]\right]$, for some $r \in \mathbb{Z}_{+}$and some simple Lie algebra $S$ (the simple Lie algebra $S$ is either finite-dimensional or one of the complete Cartan type Lie algebras $W_{N}, S_{N}, H_{N}, K_{N}$; see e.g. [G1] or [K1]). 
(b) There exists a descending family of closed ideals of $\mathfrak{L}$

$$
\mathfrak{L}=\mathfrak{J}_{0} \supset \mathfrak{J}_{1} \supset \cdots \supset \mathfrak{J}_{n} \supset \mathfrak{J}_{n+1}=0
$$

such that each quotient $\mathfrak{J}_{k} / \mathfrak{J}_{k+1}, 0 \leq k \leq n$, is either commutative or isomorphic to $\mathfrak{S}$ described in (a), in which case there are no closed ideals strictly between $\mathfrak{J}_{k}$ and $\mathfrak{J}_{k+1}$.

Lemma 5.1 tell us that we can apply Theorem 5.2 to $\left(\widehat{(\mathcal{L i e} R}^{-}\right.$. It is also easy to find out what the non-commutative quotients in (5.1) for $(\widehat{\mathcal{L i e} R)}-$ can be.

Lemma 5.2. $(\mathfrak{L i e} R)^{-}$is a Lie algebra of growth $\leq 1$ (see [GK], [K1] for the definition of growth=Gelfand-Kirillov dimension of a Lie algebra and its properties).

Proof. Let $\left\{a^{\alpha}, \alpha=1 \ldots n\right\}$ be a set of generators of the $\mathcal{A}$-module $R$. Then the elements $\partial, a_{m}^{\alpha}$ with $\alpha=1, \ldots, N, m \in \mathbb{Z}_{+}$span the Lie algebra $(\mathfrak{L i e} R)^{-}$. Consider a finite subset $X$ of this set of elements, so that for $a_{m}^{\alpha} \in X$ we have $m \leq M$, where $M$ is a positive integer. Then, due to (2.11), commutators of length $\leq n$ of elements from $X$ produce linear combinations of the $a_{m}^{\alpha}$ with $m \leq M n$ and the total number of these elements is at most $M N m$, which is a linear function in $n$, hence the growth of $(\mathfrak{L i e} R)^{-}$is at most one.

The growth of $(\mathfrak{L i e} R)^{-}$is certainly not less than the growth of the graded algebra associated to $(\widehat{\mathfrak{L i e} R)}-$ with its standard filtration, and the same applies to all subquotients. Therefore the only possible non-commutative quotients in (5.1) have an associated graded algebra of growth at most one. Since $W_{1}$ is the only complete Cartan type Lie algebra that contains a dense subalgebra of growth one, and all finite dimensional Lie algebras have growth zero, we obtain:

Lemma 5.3. The only non-commutative quotients showing up in (5.1) for the Lie algebra $(\widehat{\mathfrak{L i e} R})^{-}$are isomorphic to $\widehat{\mathfrak{V e c t} \mathbb{C}}, \mathfrak{g}$ or $\mathfrak{g}[[t]]$, where $\mathfrak{g}$ is a simple finitedimensional Lie algebra.

Lemma 5.4. If $R$ is a finite conformal algebra with no non-zero commutative ideals, then $\mathfrak{J}_{n}$ in (5.1) can be chosen to be non-commutative. 
Proof. Suppose $\mathfrak{J}_{n}$ to be commutative; then it must be central, otherwise Lemma 4.3 would give a commutative ideal of $R$.

If $\mathfrak{J}_{n}$ is central, then $\mathfrak{J}_{n-1}$ is a central extension of $\mathfrak{J}=\mathfrak{J}_{n-1} / \mathfrak{J}_{n}$ by $\mathfrak{J}_{n}$ that we may assume without loss of generality to be non-trivial. So $\mathfrak{J}_{n-1}$ is non-commutative,

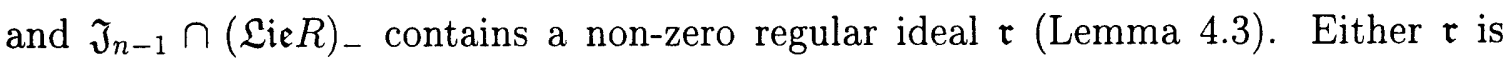
a commutative regular ideal, or it is non-commutative, in which case $[\mathfrak{r}, \mathfrak{r}] \neq 0$ is still regular (Remark 4.3) and commutative, since it is central in $(\mathfrak{L i e} R)_{-}$. But from Lemma 4.2 every commutative regular ideal corresponds to a commutative ideal of the conformal algebra $R$, thus giving a contradiction.

If $\mathfrak{J}$ is non-commutative, then by Lemma 5.3 it is isomorphic to one of the following: $\widehat{\mathfrak{V e c t} \mathbb{C}}, \mathfrak{g}$ or $\mathfrak{g}[[t]]$, where $\mathfrak{g}$ is a simple finite-dimensional Lie algebra. But those Lie algebras have no non-trivial (continuous) central extensions. So $\left[\mathfrak{J}_{n-1}, \mathfrak{J}_{n-1}\right]$ is a closed ideal isomorphic to $\mathfrak{J}$, hence minimal.

Let us now classify all surjective continuous derivations of $\mathfrak{g}[[t]]$ and $\widehat{\mathfrak{V e c t} \mathbb{C}}$.

Lemma 5.5. If $\mathfrak{S}=\mathfrak{g}[[t]]$, then $\partial=-\partial_{t}$ up to a continuous automorphism of $\mathfrak{L}$.

Proof. All continuous derivations $\partial$ of $\mathfrak{S}$ are of the form $\partial=a(t) \partial_{t}+\operatorname{ad} g(t), a(t) \in$ $\mathbb{C}[[t]], g(t) \in \mathfrak{g}[[t]]($ cf. $[\mathrm{K} 2]$, Ex. 7.4).

Surjectivity forces $a(0) \neq 0$ : since $\partial x(t)=a(t) x^{\prime}(t)+[g(t), x(t)]$, computing everything at 0 , and assuming $a(0)=0$, we get $\left.(\partial x(t))\right|_{t=0}=[g(0), x(0)]$. Since $\partial$ is surjective, all possible elements of $\mathfrak{g}$ must show up in the form $[g(0), x(0)]$ for some $x(0)$. But this is clearly impossible if $\mathfrak{g}$ is finite-dimensional. If $a(0) \neq 0$, the differential equation

$$
c(t)^{-1} c^{\prime}(t)=a(t)^{-1} \operatorname{ad} g(t) \in \operatorname{ad} \mathfrak{g}[[t]]
$$

always has a solution $c(t) \in(\operatorname{Ad} G)[[t]]$, where $G$ is the Lie group with Lie algebra $\mathfrak{g}$. But this is exactly the condition for $c(t) \partial c(t)^{-1}$ to be equal to $a(t) \partial_{t}$. Therefore $\partial$ can be conjugated to it; the automorphism mapping $\mathfrak{g}$ identically, and sending $t \mapsto-\int a(t)^{-1} d t$ clearly conjugates $a(t) \partial_{t}$ to $-\partial_{t}$. 
Lemma 5.6. If $\mathfrak{S}=\widehat{\mathfrak{V e c t} \mathbb{C}}$, then $\partial=-a d \partial_{t}$ up to a continuous automorphism.

Proof. It is a well-known fact that all continuous derivations of $\mathfrak{S}$ are inner: $\partial=\operatorname{ad} a(t) \partial_{t}, a(t) \in \mathbb{C}[[t]]$. Also $\partial$ is surjective if and only if $a(0) \neq 0$. If so, $\partial$ is conjugated to $-\partial_{t}$ by the automorphism sending $t \mapsto-\int a(t)^{-1} d t$.

Corollary 5.1. If $R$ is a simple finite conformal algebra, then $(\mathfrak{L i e} R)$ - is isomorphic to either $\mathfrak{V e c t} \mathbb{C}$ or $\mathfrak{g}[t]$, where $\mathfrak{g}$ is a finite dimensional simple Lie algebra.

Proof. $R$ has no commutative ideals so, by Lemma 5.4, $(\widehat{\mathfrak{L i e} R)}$ - has a minimal noncommutative closed ideal $\mathfrak{J}$. $\mathfrak{J}$ cannot be central hence $\mathfrak{j}=\mathfrak{J} \cap(\mathfrak{L i e} R)_{-} \neq 0$, by Proposition 4.3. $\mathfrak{j}$ must be dense in $\mathfrak{J}$ because of minimality of $\mathfrak{J}$ and it must be all

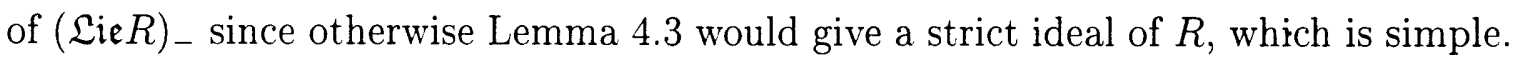

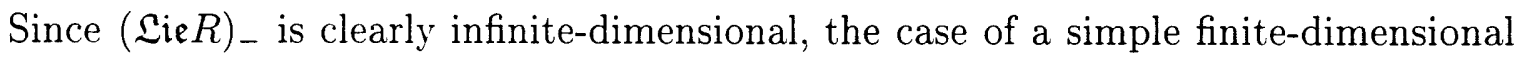
Lie algebra is ruled out.

Now, $(\mathfrak{L i e} R)_{-}$is exactly the subspace of all elements on which $\partial$ acts nilpotently, and since we know we can conjugate the action of $\partial$ to $-\partial_{t}$ (Lemmas 5.5, 5.6) it is clear that $(\mathfrak{L i e} R)_{\text {_ }}$ will be isomorphic to either $\mathfrak{V e c t} \mathbb{C}$ or $\mathfrak{g}[t]$, where $\mathfrak{g}$ is a finite dimensional simple Lie algebra.

Our classification will be proved as soon as we show that the extended annihilation algebra completely describes the conformal algebra structure.

Proposition 5.1. Let $R$ and $S$ be torsionless finite conformal algebras and $\phi:(\mathfrak{L i e} R)_{-} \rightarrow(\mathfrak{L i e} S)_{-}$be a homomorphism of topological Lie algebras compatible with the action of $\partial$. Then there is a unique homomorphism of conformal algebras $\tilde{\phi}: R \rightarrow S$ inducing $\phi$.

Proof. We are going to show that as soon as $\phi$ is a continuous linear map which is compatible with the action of $\partial$, we can find a unique $\tilde{\phi}$ inducing it. This will prove the statement since (4.9) shows how to recover the conformal algebra structure from the Lie algebra structure of the annihilation algebra. Uniqueness of $\tilde{\phi}$ will then show that $\tilde{\phi}$ is a homomorphism of $\mathcal{A}$-modules. 
Since $S$ has no torsion, by Lemma 4.1 for every choice of $r \in R$ there is at most one $s \in S$ such that $s_{2}=\phi\left(r_{\imath}\right)$ for every $i$.

Let us fix a basis $\left\{s^{i}\right\}$ of $S$ and consider an element $r \in R$. Because of the compatibility of $\phi$ with the action of $\partial$, there always exist numbers $c_{\jmath}^{i}$ such that

$$
\phi\left(r_{n}\right)=\sum_{j=0}^{n} \sum_{i}(-1)^{n-j} \frac{n !}{j !} c_{n-j}^{2} s_{\jmath}^{i}
$$

for every $n \in \mathbb{Z}_{+}$. Then set

$$
\tilde{\phi}(r)=\sum_{\jmath=0}^{\infty} c_{\jmath}^{i} \partial^{j} s^{i}
$$

The formal element $\tilde{\phi}(r)$ is such that $(\tilde{\phi}(r))_{n}=\phi\left(r_{n}\right)$ for all $n \in \mathbb{Z}_{+}$. Because of continuity of $\phi$, for every $r \in R, \phi\left(r_{\imath}\right)$ lies in $\mathfrak{L}_{1}$ for $i>>0$ (see (4.6)). But this forces $\tilde{\phi}(r)$ to be polynomial in $\partial$ and therefore to belong to $S$. Then, due to uniqueness of $\tilde{\phi}(r)$ the map $r \mapsto \tilde{\phi}(r)$ is $\mathcal{A}$-linear. Moreover $\tilde{\phi}$ clearly induces $\phi$.

We are now able to classify all finite simple conformal algebra.

Proof of Theorem 5.1. We already know that Vir and $\operatorname{Cur} \mathfrak{g}$, where $\mathfrak{g}$ is a finite dimensional simple Lie algebra, are simple conformal algebras. We want to show they are the only ones.

Let $R$ be a simple conformal algebra. Then Corollary 5.1 shows $(\mathfrak{L i e} R)_{-}$is isomorphic to either $\mathfrak{V e c t} \mathbb{C}$ or $\mathfrak{g}[t]$ with the topology induced by the standard filtrations and that $\partial$ can be assumed to act as $-\partial_{t}$. So we get $\partial$-compatible continuous isomorphisms between $(\mathfrak{L i e} R)_{-}$and either $(\mathfrak{L i e} V i r)_{-}$or $(\mathfrak{L i e} C u r \mathfrak{g})_{-}$, resulting by Proposition 5.1 in isomorphisms of $R$ with either Vir or $C u r \mathfrak{g}$. This concludes the proof.

The correspondence between conformal algebras and formal distribution Lie algebras (Remark 2.3) implies the following corollary of Theorem 5.1.

Corollary 5.2. A finite simple (i.e. without non-trivial regular ideals) formal distribution Lie algebra is isomorphic either to the Virasoro Lie algebra or to a quotient of a current Lie algebra $\mathfrak{g}\left[t, t^{-1}\right]$ with $\mathfrak{g}$ a finite-dimensional simple Lie algebra. 


\section{Chapter 6}

\section{The Killing form}

The next step towards a complete structure theory of conformal algebras is the classification of semi-simple algebras. In the theory of finite-dimensional Lie algebras we can rely on the inestimable use of the Killing form. It is evident we need an analogous notion in the conformal algebra case, but the very notion of trace of a conformal linear map is ill-defined. Life would be much easier if we were handling the usual $\mathcal{A}$-linear maps.

Definition 6.1. Let $M$ be a finite rank $\mathcal{A}$-module and $T: M \rightarrow M$ an $\mathcal{A}$-linear map. Let $\mathcal{K}=\mathbb{C}(\partial)$ be the field of fractions of $\mathcal{A}$ and let $M_{\mathcal{K}}=\mathcal{K} \otimes_{\mathcal{A}} M$. Then $T$ extends by linearity to a $\mathcal{K}$-linear map $M_{\mathcal{K}} \rightarrow M_{\mathcal{K}}$ and the trace of $T$ on $M$ is defined as $\operatorname{Tr}_{M} T=\operatorname{Tr}_{M_{\mathcal{K}}} T_{\mathcal{K}}$.

Remark 6.1. This trace inherits all standard properties of a trace on a vector space. In particular $\operatorname{Tr}_{M} A B=\operatorname{Tr}_{M} B A$. Furthermore, if $M$ is a free module, then $\operatorname{Tr}_{M} T$ is equal to the sum of elements on the diagonal of any matrix representation of $T$. Also $\operatorname{Tr}_{M} T=\operatorname{Tr}_{M / \text { Tor }}{ }_{M} T$.

In spite of the fact that conformal linear maps are not $\mathcal{A}$-linear, one obviously has:

Proposition 6.1. Let $\phi^{i}$ be conformal linear maps, $\lambda_{2}$ indeterminates. Then the composition $\phi_{\lambda_{1}}^{1} \phi_{\lambda_{2}}^{2} \ldots \phi_{\lambda_{n}}^{n}$ is $\mathcal{A}$-linear whenever $\sum_{i} \lambda_{\imath}=0$. 
In this way, the trace of the composition of conformal linear maps is well-defined as soon as the $\lambda$ 's add up to 0 . The analogue of the commutativity property of trace in this case is:

Proposition 6.2. Let $M$ be an $\mathcal{A}$-module of finite rank, and for $p(\partial) \in \mathcal{A}$ let $s_{c} p(\partial)=p(\partial+c)$. If $\phi^{2} \in$ Cend $M$, then one has

$$
\operatorname{Tr}_{M} \phi_{\lambda_{1}}^{1} \phi_{\lambda_{2}}^{2} \ldots \phi_{\lambda_{n}}^{n}=s_{\lambda_{1}} \operatorname{Tr}_{M} \phi_{\lambda_{2}}^{2} \ldots \phi_{\lambda_{n}}^{n} \phi_{\lambda_{1}}^{1}
$$

Proof. We may assume that $M$ is a free $\mathcal{A}$-module (see Remark 6.1). Choose an $\mathcal{A}$-basis $\left\{m^{i}\right\}$ of $M$. Then there is a unique conformal linear map $I: M \rightarrow M$ such that $I_{\lambda} m^{i}=m^{i}$ for all $i$. The composition $I_{\lambda} I_{-\lambda}$ is always the identity.

Let $T$ be an $\mathcal{A}$-linear map on $M$. Then $I_{\lambda} T I_{-\lambda}$ is $\mathcal{A}$-linear and we have:

$$
\operatorname{Tr}_{M} I_{\lambda} T I_{-\lambda}=s_{\lambda} \operatorname{Tr}_{M} T
$$

as can be easily shown by adding up elements on the diagonal of the matrix representation of the composition. Proposition follows now by noticing that Remark 6.1 implies:

$$
\begin{aligned}
& \operatorname{Tr}_{M} \phi_{\lambda_{1}}^{1} \phi_{\lambda_{2}}^{2} \ldots \phi_{\lambda_{n}}^{n}= \\
& \quad=\operatorname{Tr}_{M}\left(\phi_{\lambda_{1}}^{1} I_{-\lambda_{1}}\right) I_{\lambda_{1}} \phi_{\lambda_{2}}^{2} \ldots \phi_{\lambda_{n}}^{n}=\operatorname{Tr}_{M} I_{\lambda_{1}} \phi_{\lambda_{2}}^{2} \ldots \phi_{\lambda_{n}}^{n} \phi_{\lambda_{1}}^{1} I_{-\lambda_{1}}
\end{aligned}
$$

We are now able to define our "Killing form".

Definition 6.2. Let $R$ be a finite conformal algebra. The Killing form of $R$ is the $\mathbb{C}$-bilinear pairing

$$
(x \mid y)_{\lambda}=\operatorname{Tr}_{R}(\operatorname{ad} x)_{\lambda}(\operatorname{ad} y)_{-\lambda} .
$$

Due to (6.1), the Killing form satisfies

$$
(x \mid y)_{\lambda}=s_{\lambda}(y \mid x)_{-\lambda} .
$$


It is also clear by definition that

$$
(\partial x \mid y)_{\lambda}=-(x \mid \partial y)_{\lambda}=-\lambda(x \mid y)_{\lambda} .
$$

Example 6.1. Let $R=V i r=\mathcal{A} L$. Then the Killing form is

$$
(L \mid L)_{\lambda}=(\partial-\lambda)(\partial+2 \lambda) \text {. }
$$

Example 6.2. If $R=C u r \mathfrak{g}, g, h \in \mathfrak{g} \subset C u r \mathfrak{g}$ then

$$
(g \mid h)_{\lambda}=\kappa(g \mid h),
$$

where $\kappa$ is the Killing form of the Lie algebra $\mathfrak{g}$.

For a subalgebra $I$ of $R$ set $I^{\perp}=\left\{x \in R \mid(x \mid I)_{\lambda}=0\right\}$. Due to (6.3) we get $I^{\perp}=\left\{x \in R \mid(I \mid x)_{\lambda}=0\right\}$, and due to (6.4), $I^{\perp}$ is an $\mathcal{A}$-submodule. The following example shows that $I^{\perp}$ does not need to be an ideal of $R$ even if $I$ is.

Example 6.3. Let $R$ be the semi-direct sum of $\operatorname{Vir}$ and $C u r \mathfrak{g}$, where $\mathfrak{g}$ is semisimple, given by $L_{\lambda} r=d_{\lambda}^{L} r$ as in Example 3.4. Then

$$
(g \mid h)_{\lambda}=\kappa(g \mid h), \quad(L \mid L)_{\lambda}=(\partial-\lambda)(\partial+2 \lambda), \quad(L \mid g)_{\lambda}=0
$$

for every $g, h \in \mathfrak{g}$. Therefore, Cur $\mathfrak{g}$ is an ideal of $R$, Cur $\mathfrak{g}^{\perp}=$ Vir but Vir is not an ideal.

What is instead true is that $I^{\perp}$ is always a subalgebra.

Proposition 6.3. Let $R$ be a conformal algebra and $I \subset R$ an ideal. Then $I^{\perp}$ is a subalgebra of $R$.

Proof. Let $a \in I, b, c \in I^{\perp}$. Then, since $I$ is closed under $\lambda$-bracket with every element of $R$, we have:

$$
\left(\left[a_{\lambda} b\right] \mid c\right)_{\lambda+\mu}=\left(\left[a_{\lambda} c\right] \mid b\right)_{\mu}=\left(c \mid\left[a_{\lambda} b\right]\right)_{-\lambda-\mu}=0 .
$$


Vanishing of the first term implies

$$
\operatorname{Tr}_{R} \text { ad } a_{\lambda} \text { ad } b_{\mu} \text { ad } c_{-\lambda-\mu}=\operatorname{Tr}_{R} \text { ad } b_{\mu} \text { a } 1 a_{\lambda} \text { ad } c_{-\lambda-\mu}
$$

while that of the other two shows

$$
\begin{aligned}
& \operatorname{Tr}_{R} \operatorname{ad} a_{\lambda} \operatorname{ad} c_{-\lambda-\mu} \text { ad } b_{\mu}= \\
& \quad=\operatorname{Tr}_{R} \operatorname{ad} c_{-\lambda-\mu} \operatorname{ad} a_{\lambda} \operatorname{ad} b_{\mu}=\operatorname{Tr}_{R} \operatorname{ad} c_{-\lambda-\mu} \operatorname{ad} b_{\mu} \operatorname{ad} a_{\lambda} .
\end{aligned}
$$

We want to show that $\left(a \mid\left[b_{\mu} c\right]\right)_{\lambda}=0$, that is:

$$
\operatorname{Tr}_{R} \text { ad } a_{\lambda} \text { ad } b_{\mu} \text { ad } c_{-\lambda-\mu}=\operatorname{Tr} \operatorname{ad} a_{\lambda} \operatorname{ad} c_{-\lambda-\mu} \operatorname{ad} b_{\mu}
$$

But we know from Proposition 6.2 and (6.6) that

$$
\begin{aligned}
& \operatorname{Tr}_{R} \operatorname{ad} a_{\lambda} \operatorname{ad} c_{-\lambda-\mu} \text { ad } b_{\mu}= \\
& \quad=s_{\lambda} \operatorname{Tr}_{R} \operatorname{ad} c_{-\lambda-\mu} \text { ad } b_{\mu} \operatorname{ad} a_{\lambda}=s_{\lambda} \operatorname{Tr}_{R} \operatorname{ad} a_{\lambda} \operatorname{ad} c_{-\lambda-\mu} \operatorname{ad} b_{\mu} .
\end{aligned}
$$

This shows that the polynomial $\operatorname{Tr}_{R}$ ad $a_{\lambda} \operatorname{ad} c_{-\lambda-\mu}$ ad $b_{\mu}$ is independent of $\partial$. The rest of the proof follows easily. Using Proposition 6.2, independence of $\operatorname{Tr}_{R}$ ad $a_{\lambda} \operatorname{ad} c_{-\lambda-\mu}$ ad $b_{\mu}$ with respect to $\partial$, and (6.5) we obtain:

$$
\begin{aligned}
& \operatorname{Tr}_{R} \operatorname{ad} a_{\lambda} \operatorname{ad} c_{-\lambda-\mu} \text { ad } b_{\mu}= \\
& \quad=\operatorname{Tr}_{R} \operatorname{ad} b_{\mu} \operatorname{ad} a_{\lambda} \operatorname{ad} c_{-\lambda-\mu}=\operatorname{Tr}_{R} \text { ad } a_{\lambda} \operatorname{ad} b_{\mu} \operatorname{ad} c_{-\lambda-\mu}
\end{aligned}
$$

establishing (6.7).

Remark 6.2. All properties of the Killing form extend to the trace form in any finite $R$-module.

The subalgebra $I^{\perp}$ does not even need to be a complementary submodule to $I$. Nevertheless one has:

Proposition 6.4. Let $I \subset R$ be an ideal isomorphic to either $V i r$ or $C u r \mathfrak{g}$, for a simple finite-dimensional Lie algebra $\mathfrak{g}$. Then $R=I^{\perp} \oplus I$ as $\mathcal{A}$-modules. 
In order to prove this statement, we need a classification of all conformal derivations (see Definition 3.2) of Vir and Cur $\mathfrak{g}$.

Lemma 6.1. Every conformal derivation d of the conformal algebra Vir is inner.

Proof. Let us assume that $d_{\lambda} L=a(\partial, \lambda) L$ where $a \in \mathbb{C}[\partial, \lambda]$. We write $a=\sum_{\imath=1}^{n} a_{i}(\lambda) \partial^{i}$. If $d$ is a conformal derivation, it needs to satisfy $d_{\lambda}\left[L_{\mu} L\right]=\left[L_{\mu}\left(d_{\lambda} L\right)\right]^{\circ}+\left[\left(d_{\lambda} L\right)_{\lambda+\mu} L\right]$. This is equivalent to saying that

$$
(\partial+\lambda+2 \mu) a(\partial, \lambda) L=a(\partial+\mu, \lambda)(\partial+2 \mu) L+a(-\lambda-\mu, \lambda)(\partial+2 \lambda+2 \mu) L .
$$

Assuming all $a_{\imath}$ 's are non-zero and equating terms of degree $n$ in $\partial$ in both sides, we get, if $n>1,(\lambda-n \mu) a_{n}(\lambda)=0$. This shows that $a_{n}(\lambda)=0$, a contradiction. Therefore the degree of $a(\partial, \lambda)$ in $\partial$ can be at most one. In this case, substituting into the derivation requirement, one gets $a_{0}(\lambda)=2 \lambda a_{1}(\lambda)$, hence $d_{\lambda} L=a_{1}(\lambda)(\partial+2 \lambda) L$, which is an inner derivation induced by the element $a_{1}(-\partial) L$.

Lemma 6.2. Let $\mathfrak{g}$ be a simple finite-dimensional Lie algebra. Then every conformal derivation $d$ of Cur $\mathfrak{g}$ is of the form $p(\partial) d^{L}+d$ where $d$ is inner and $d^{L}$ is as in Example 3.4.

Proof. Set $d_{\lambda} g=\sum_{\imath \in \mathbb{Z}_{+}} \partial^{\imath} d_{\lambda}^{2}(g)$, for every $g \in \mathfrak{g}$, where $d_{\lambda}^{i}$ are $\mathbb{C}$-linear maps of $\mathfrak{g}$ to $\mathbb{C}[\lambda] \otimes \mathfrak{g}$. Then (3.5) tells us that

$$
\sum_{\imath} \partial^{i} d_{\lambda}^{2}([g, h])=\sum_{\imath}\left((-\lambda-\mu)^{2}\left[d_{\lambda}^{2} g, h\right]+(\partial+\mu)^{2}\left[g, d_{\lambda}^{2} h\right]\right)
$$

Setting $\mu=0$, switching the roles of $g$ and $h$ and adding up, one gets:

$$
\sum_{\imath} \partial^{\imath}\left(\left[g, d_{\lambda}^{i} h\right]+\left[h, d_{\lambda}^{i} g\right]\right)=\sum_{i}(-\lambda)^{i}\left(\left[g, d_{\lambda}^{i} h\right]+\left[h, d_{\lambda}^{i} g\right]\right)
$$

The right hand side does not depend on $\partial$, hence all coefficients of non-zero powers of $\partial$ must be 0 . This means that $\left[d_{\lambda}^{2} g, h\right]+\left[d_{\lambda}^{i} h, g\right]=0$ for every $i>0$ and every $g, h \in \mathfrak{g}$. If $\mathfrak{g}$ is simple, such a linear map can only be a multiple of the identity, as 
next lemma will show, hence $d$ must be of the form:

$$
d_{\lambda} g=d_{\lambda}^{0}(g)+p(\partial, \lambda) g
$$

for every $g \in \mathfrak{g}$. But $d$ is a conformal derivation, so if $n>1$ and $p(\partial, \lambda)=\sum_{i=0}^{n} p_{\imath}(\lambda) \partial^{i}$, equating $n-1$ degree terms in (3.5), we get $n \mu \dot{p}_{n}(\mu)=0$. This proves that $p$ is of degree at most one in $\partial$.

Let $d_{\lambda}^{\prime} g=p_{1}(\lambda)(\partial+\lambda) g$, i.e. $d^{\prime}=p_{1}(-\partial) d^{L}$. This is a conformal derivation of $\mathfrak{g}$ and the action of $d-d^{\prime}$ on $\mathfrak{g}$ is independent of $\partial$. Hence, by (6.8), $d_{\lambda}-d^{\prime}$ is an ordinary derivation from $\mathfrak{g}$ to $\mathbb{C}[\lambda] \otimes \mathfrak{g}$ and is therefore an inner conformal derivation, proving the lemma.

Lemma 6.3. Let $\mathfrak{g}$ be a simple finite-dimensional Lie algebra, and $T: \mathfrak{g} \rightarrow \mathfrak{g}$ a linear map. If $[T x, y]=[x, T y]$ for all $x, y \in \mathfrak{g}$ then $T$ is a multiple of the identity map.

Proof. Let $\mathfrak{g}=\bigoplus_{\alpha} \mathfrak{g}_{\alpha}$ be a root space decomposition with respect to a Cartan subalgebra $\mathfrak{h}=\mathfrak{g}_{0}$. Since $[T x, x]=0$, if $x \in \mathfrak{h}$ is a regular element, then $T x$ lies in the centralizer of $x$, hence in $\mathfrak{h}$. This shows that $T \mathfrak{h} \subset \mathfrak{h}$. This means that $T$ preserves the root decomposition of $\mathfrak{g}$ modulo elements in $\mathfrak{h}$. In fact if $x \in \mathfrak{g}_{\alpha}$, then $[T h, x]=[h, T x], h \in \mathfrak{h}$, and since the left hand side is a multiple of $x$, the right hand side must be too, showing that $T x$ is a multiple of $x$ plus some element from $\mathfrak{h}$ : $T x=c_{\alpha} x+h(x)$.

Let now $x \in \mathfrak{g}_{\alpha}, y \in \mathfrak{g}_{\beta}$. From $[T x, y]=[x, T y]$ we obtain

$$
c_{\alpha}[x, y]+[h(x), y]=c_{\beta}[x, y]+[x, h(y)]
$$

When $\alpha$ and $\beta$ are unequal roots, this means

$$
\left(c_{\alpha}-c_{\beta}\right)[x, y]=0,[h(x), y]=0,[x, h(y)]=0
$$

Since $\mathfrak{g}$ is simple, its Dynkin diagram is connected, and this allows us to show that all $c_{\alpha}=c_{\beta}=c$ are the same for all roots $\alpha, \beta$. But $[h(x), y]=0$ for all root 
vectors $y$ gives $h(x)=0$. Hence $T$ equals $c$ Id on all $\mathfrak{g}_{\alpha}, \alpha \neq 0$. It is left to show that $T$ equals $c$ Id also on $\mathfrak{h}$. But $[T h, x]=[h, T x]$ gives $[T h-c h, x]=0$ as soon as $x$ is a root vector. This tells us that $T h=c h$ also for $h \in \mathfrak{h}$.

Proof of Proposition 6.4. Let $a \in R, j \in J$. Then in taking $(a \mid j)_{\lambda}$ it is enough to consider the trace over $J$ since this is an ideal. Element $a$ acts on $J$ via a conformal derivation. But any conformal derivation of $V i r$ is inner, and any non-inner conformal derivation of $\operatorname{Cur} \mathfrak{g}$ is as in Lemma 6.2. Any element acting as $d^{L}$ on $C u r \mathfrak{g}$ has a zero Killing product with it.

In any case $j \mapsto(a \mid j)_{\lambda}$ is equal to $j \mapsto(i \mid j)_{\lambda}$ for some $i \in J$, and all $j \in J$. This, and the fact that the Killing form is non-degenerate on simple algebras (which we need in order to show uniqueness in the choice of $i$ ), show that $J+J^{\perp}$ is a direct sum decomposition.

Remark 6.3. One can prove the proposition when the ideal $J$ is isomorphic to the Virasoro conformal algebra without using the Killing form. Indeed, let $C$ be the centralizer of this ideal. It will be an ideal, and since all derivation of $J$ are inner and its center is trivial, every element $x$ in our conformal algebra admits a unique decomposition $x=x_{C}+x_{J}$ where $x_{C}$ lies in the $C$ and $x_{J}$ lies in $J$.

Thus, $C+J$ is a direct sum decomposition, and $C$ is shown to be a complementary ideal, and not only a subalgebra. This argument is applicable any time $J$ is centerless and has no outer derivations. 


\section{Chapter 7}

\section{Semi-simple conformal algebras}

We have all the tools we need in order to attack the problem of classification of semi-simple conformal algebras, now. Let us start by giving the basic definitions.

Definition 7.1. Let $R$ be a conformal algebra, $I$ and $J$ its ideals. The bracket $[I \cdot J]$ of these is the subspace of $R$ that is spanned by all products $i_{(n)} j$ with $i \in I$, $j \in J, n \in \mathbb{Z}_{+}$. Note that this is an $\mathcal{A}$-module due to (C2) and an ideal due to (C4). In other words $[I \cdot J]=\left\langle\left[I_{\lambda} J\right]\right\rangle$ (see Lemma 3.1).

The derived conformal algebra of $R$ is $R^{\prime}=[R \cdot R]$. We set $R^{(1)}=R^{\prime}$, $R^{(n+1)}=\left(R^{(n)}\right)^{\prime}, n \geq 1$. Then $R$ is a solvable conformal algebra if $R^{(n)}=0$ for

some $n \geq 1$. An ideal $I \subset R$ is solvable if it is solvable as a conformal algebra. If $I$ is an ideal of $R$, we define $I^{1}=I$ and $I^{n+1}=\left[R \cdot I^{n}\right], n \geq 1 . R$ is a nilpotent conformal algebra if $R^{n}=0$.

A conformal algebra $R$ is semi-simple if it has no non-zero solvable ideals.

Recall that by (2.11) and (4.9) (see also Remark 4.3) we have for any two ideals $I$ and $J$ of a conformal algebra $R$ :

$$
(\mathfrak{L} \mathfrak{i} e[I \cdot J])_{-}=\left[(\mathfrak{L i e} I)_{-},(\mathfrak{L i e} J)_{-}\right] .
$$

Lemma 7.1. A conformal algebra $R$ is solvable (resp. nilpotent) if and only if its annihilation algebra $(\mathfrak{L i e} R)_{-}$is. 
Proof. We proceed as in Lemma 4.2. $R$ is solvable (resp. nilpotent) if and only if $R / \operatorname{Tor} R$ is, so we will assume $R$ to be torsionless. Due to (7.1), we have: $\left(\mathfrak{L i} \mathfrak{e} R^{(n)}\right)_{-}=(\mathfrak{L} \mathfrak{i} R)_{-}^{(n)},\left(\mathfrak{L i} \mathfrak{i} R^{n}\right)_{-}=(\mathfrak{L} \mathfrak{i} R)^{n}$. The result follows form the fact that $J \subset R,(\mathfrak{L i e} J)_{-}=0$ implies $J=0$, when $R$ is torsionless (see Lemma 4.1).

Remark 7.1. Here and further, we have denoted the subspace $\mathfrak{j}$ of $(\mathfrak{L i e} R)_{-}$spanned by all Fourier coefficients of members from an ideal $J$ of a conformal algebra $R$ by $(\mathfrak{L i e} J)_{-}$. Even though the inclusion of an ideal $J$ in a conformal algebra $R$ is clearly injective, the map it induces at the level of annihilation algebras might have a nontrivial kernel. However this kernel $\mathfrak{k}$ must be central in $(\mathfrak{L} \mathfrak{i} J)_{\text {. }}$. In fact, if $\mathfrak{k}$ were not central, Lemma 4.3 would locate a non-zero regular subideal of $\mathfrak{k}$, which amounts to finding a non-zero ideal $K$ in $J$ that is mapped to 0 by the inclusion $J \rightarrow R$, thus obtaining a contradiction. Since one can usually reconstruct the algebraic properties of the ideal $J$ from $\mathfrak{j}$, this abuse of notation should cause no confusion.

Lemma 7.2. If $R$ is a finite semi-simple conformal algebra, then any minimal ideal of $R$ is simple.

Proof. Let $I$ be a minimal ideal of $R$. Then $(\widehat{\mathfrak{L i e} I)}$ - must contain a minimal closed ideal $\mathfrak{I}$ of $\left(\widehat{\mathfrak{L i e} R)^{-}}\right.$due to finiteness of descending chains of ideals [G], and we can assume it to be non-commutative by arguing as in Lemma 5.4. Since $\mathfrak{I}$ is not central, it has a non-zero intersection with $(\mathfrak{L} \mathfrak{i} e R)_{-}$, which must contain a non-zero regular ideal $\mathfrak{i}$ by Lemma 4.3. Minimality of $I$ then shows that $\mathfrak{i}$ equals $(\mathfrak{L i e} I)_{-}$. As in Corollary $5.1, \mathfrak{i}$ is the space of all elements in the non-commutative closed ideal $\mathfrak{I}$ on which $\partial$ acts nilpotently, and by Lemmas 5.5 and 5.6 it is clear that $\mathfrak{i}$ must be isomorphic to either $\mathfrak{V e c t} \mathbb{C}$ or $\mathfrak{g}[t]$, where $\mathfrak{g}$ is a finite-dimensional simple Lie algebra, and that $\partial$ can be assumed to act on $\mathfrak{i}$ as $-\partial_{t}$.

This allows us to give a continuous embedding $\phi$ of $\mathfrak{V e c t} \mathbb{C}$ (resp. $\mathfrak{g}[t])$ in $\mathfrak{L i e} R$ which is compatible with the action of $\partial$. Proposition 5.1 then shows that, since $I$ is clearly torsionless, there is an embedding $\tilde{\phi}$ of $\operatorname{Vir}$ (resp. Cur $\mathfrak{g}$ ) in $R$ which is an isomorphism onto $I$. Therefore $I$ is simple as a conformal algebra.

The main theorem we are going to prove is the following. 
Theorem 7.1. Any finite semi-simple conformal algebra can be uniquely decomposed in a finite direct sum of conformal algebras each of which is isomorphic to one of the following:

(a) Vir

(b) Cur $\mathfrak{g}$, where $\mathfrak{g}$ is a simple finite-dimensional Lie algebra

(c) The semi-direct sum of Vir and Cur $\mathfrak{g}$, where $\mathfrak{g}$ is a semi-simple finitedimensional Lie algebra (Example 3.4).

Proof. Let $R$ be a semi-simple conformal algebra. We prove the theorem by induction on $\operatorname{rk} R$.

If $R$ has an ideal isomorphic to Vir then by Remark 6.3 it has a complementary ideal that centralizes it. Therefore it splits up in a direct sum decomposition. Hence, we may assume that $R$ does not contain any ideal isomorphic to $V i r$.

Consider all minimal ideals $I_{1}, I_{2}, \ldots, I_{n}$ of $R$. They are simple by Lemma 7.2. We assumed that no ideal of $R$ is isomorphic to Vir, hence $I=I_{1}+I_{2}+\ldots+I_{n}$ is an ideal of $R$ isomorphic to $\operatorname{Cur} \mathfrak{g}$, where $\mathfrak{g}$ is a semi-simple finite-dimensional Lie algebra. The centralizer $C$ of $I$ is an ideal of $R$ intersecting $I$ trivially. Since $C$ must contain a minimal subideal, $C=0$.

Consider the orthocomplement $I^{\perp}$ of $I$ with respect to the Killing form. Proposition 6.4 also applies (by induction) to direct sums of simple algebras, so $R=I^{\perp} \oplus I$ as $\mathcal{A}$-modules.

If $I^{\perp}$ had a commutative ideal $A$, it may act on every $I_{i}$ (hence on all of $I$ ) only by inner derivations (by Lemma 6.2), hence since $C=0$, we get $A=0$. Thus, $I^{\perp}$ is semi-simple and we can apply the inductive assumption. Note that $I^{\perp}$ contains no currents (i.e. elements $a$ such that $a_{\lambda} a=0$ ), otherwise $C \neq 0$. So $I^{\perp}$ is a direct sum of (its) ideals isomorphic to Vir.

The action of all such ideals on $I_{i}$ is either zero or the standard one (Example 3.4) since they lie inside $I^{\perp}$ and no two Vir's can act in a non-zero way on the same $I_{i}$ because of [CK], Proposition 3.1, or Proposition 8.1 below.

As in the case of simple conformal algebras, Theorem 7.1 implies 
Corollary 7.1. A finite semi-simple formal distribution Lie algebra can be uniquely decomposed in a finite direct sum of formal distribution Lie algebras each of which is isomorphic either to a finite simple formal distribution Lie algebra (classified by Corollary 5.2), or to the semi-direct sum of the Virasoro Lie algebra with a current Lie algebra $\mathfrak{g}\left[t, t^{-1}\right]$, where $\mathfrak{g}$ is a finite-dimensional semi-simple Lie algebra. 


\section{Chapter 8}

\section{Representation theory}

Definition 8.1. Let $R$ be a conformal algebra. Then an $\mathcal{A}$-module $V$ is a representation of $R$, or an $R$-module, if for every $r \in R$ a conformal linear map $r: V \rightarrow V$ is defined such that $(r, s \in R, v \in V)$ :

$$
r_{\lambda}\left(s_{\mu} v\right)-s_{\mu}\left(r_{\lambda} v\right)=\left[r_{\lambda} s\right]_{\lambda+\mu} v, \quad(\partial r)_{\lambda} v=-\lambda r_{\lambda} v .
$$

As before, we will often write $r_{\lambda}=\sum_{n \in \mathbb{Z}_{+}} \lambda^{(n)} r_{n}, r_{n} \in \operatorname{End} V$. An $R$-module $V$ is called finite if it is a finitely generated $\mathcal{A}$-module. Note also that a representation of $R$ in an $\mathcal{A}$-module $V$ is the same as a homomorphism (of conformal algebras) $R \rightarrow g c V$.

Remark 8.1. Let $R$ be a finite conformal algebra with a faithful representation $V=\mathcal{A} v$ which is free of rank one, i.e. we have an injective homomorphism of conformal algebras $R \rightarrow g c_{1}$. By (3.7) an element $\sum_{n \in \mathbb{Z}_{+}} p_{n}(\partial) T^{n}$ generates an infinite rank subalgebra of $g c_{1}$ as soon as $p_{i} \neq 0$ for some $i>1$. Therefore, the image of $R$ in $g c_{1}$ must lie in the subalgebra $\mathcal{A} T^{0}+\mathcal{A} T^{1}$. This implies that the only finite conformal algebras that have a faithful representation which is free of rank one as an $\mathcal{A}$-module are subalgebras of the standard semi-direct sum (see Example 3.4) of $V$ ir and a commutative current algebra $\mathrm{Cur} \mathbb{C}$.

It is immediate to see that a representation of a conformal algebra $R$ in $V$ is the same as a representation of the Lie algebra $(\mathfrak{L i e} R)^{-}$in $V$ satisfying the local 
nilpotency condition

$$
r_{n^{\prime}}=0 \text { for } n \text { sufficiently large, } r \in R, v \in V \text {. }
$$

A $(\mathfrak{L} \mathfrak{i e} R)^{-}$-module satisfying this condition is called conformal.

This turns out to be a more convenient language for the study of representations of conformal algebras, using which Cheng and $\mathrm{Kac}$ [CK] classified all irreducible representations of $V i r, C u r \mathfrak{g}$ and their non-trivial semi-direct sum.

We shall often write $R \cdot V$ for $\sum_{\jmath \in \mathbb{Z}_{+}} R_{\jmath} V$. A representation $V$ of the conformal algebra $R$ is called trivial.if $R \cdot V=0$. Recall that a finite-dimensional Lie algebra $\mathfrak{g}$ is called reductive is it is a direct sum of a semi-simple Lie algebra $\mathfrak{g}^{\prime}$ and a commutative Lie algebra $\mathfrak{a}$.

Theorem 8.1. Let $\mathfrak{g}=\mathfrak{g}^{\prime} \oplus \mathfrak{a}$ be a reductive Lie algebra. Then any non-trivial finite rank irreducible representation of Cur $\mathfrak{g}$ is of the form $V(U, \phi)=\mathcal{A} \otimes U$, where $U$ is an irreducible finite-dimensional $\mathfrak{g}$-module, $\mathfrak{a} \ni a \mapsto \phi_{a} \in \mathbb{C}[\lambda]$ is a linear map such that $a=\phi_{a}(0)$ on $U$ and either $U$ is non-trivial or $\phi \neq 0$, and the action of Cur $\mathfrak{g}$ on $V(U, \phi)$ is the unique one extending

$$
g_{\lambda} u=g . u, a_{\lambda} u=\phi_{a}(\lambda) u
$$

where $g \in \mathfrak{g}^{\prime} \subset$ Cur $\mathfrak{g}, a \in \mathfrak{a} \subset$ Cur $\mathfrak{g}, u \in U$ and $g . u$ denotes the action of $g \in \mathfrak{g}^{\prime}$ on the $\mathfrak{g}$-module $U$.

Theorem 8.2. Any non-trivial representation $V$ of $V$ ir that is free of rank one as an $\mathcal{A}$-module is of the form

$$
L_{\lambda} v=(c+\partial+\Delta \lambda) v
$$

where $\Delta, c \in \mathbb{C}$ and $v$ is a free generator of $V$. This representation is irreducible if and only if $\Delta$ is non-zero, and all irreducible representations of Vir are of this kind.

Theorem 8.3. Any non-trivial irreducible representation of the semi-direct sum of 
Vir and Cur $\mathfrak{g}$, where $\mathfrak{g}$ is a non-zero reductive Lie algebra, is of the form $V(U)=\mathcal{A} \otimes U$, where $U$ is an irreducible finite-dimensional $\mathfrak{g}$-module, and the action is the unique one extending

$$
g_{\lambda} u=g \cdot u, \quad L_{\lambda} u=(c+\partial+\Delta \lambda) u
$$

where $c, \Delta \in \mathbb{C}, g \in \mathfrak{g} \subset C u r \mathfrak{g}, u \in U$, and $U$ is a non-trivial $\mathfrak{g}$-module if $\Delta=0$.

Proposition 8.1. Let $R$ be a direct sum of conformal algebras isomorphic to one of the following:

(i) Cur $\mathfrak{g}$, where $\mathfrak{g}$ is a non-zero reductive Lie algebra;

(ii) the standard semi-direct sum of Vir and Cur $\mathfrak{g}$, where $\mathfrak{g}$ is reductive or 0 .

Suppose that $R$ has a finite faithful irreducible representation. Then $R$ is either of type (i) or of type (ii) where $\mathfrak{g}$ has at most one-dimensional center. Finite irreducible representations of $R$ are described by Theorems 8.1-8.3.

The proof of these results is the same as in $[\mathrm{CK}]$. It relies on the following key lemma, that we will occasionally use later.

Lemma 8.1. [CK] Let $\mathfrak{L}$ be a Lie algebra, with a distinguished element $\partial$ and a descending sequence of subspaces

$$
\mathfrak{L} \supset \mathfrak{L}_{0} \supset \mathfrak{L}_{1} \supset \ldots
$$

such that $\left[\partial, \mathfrak{L}_{n}\right]=\mathfrak{L}_{n-1}$ for all $n>0$. Let $V$ be a $\mathfrak{L}$-module and let $V_{n}=\left\{v \in V \mid \mathfrak{L}_{n} v=0\right\}$; suppose that $V_{n} \neq 0$ for $n$ sufficiently large, and set $N$ to be minimal such that $V_{N} \neq 0$. Then, provided that $N \geq 1, \mathcal{A} V_{N}=\mathcal{A} \otimes V_{N}$. In particular, $V_{N}$ is a finite-dimensional vector space (over $\mathbb{C}$ ) if $N \geq 1$ and $V$ is a finitely generated $\mathcal{A}$-module.

Clearly, if $\mathfrak{L}=(\mathfrak{L i \mathfrak { e }} R)^{-}$and $V$ is a module over $R$ (i.e. a conformal module over $\mathfrak{L}$ ), the conditions of Lemma 8.1 are satisfied (cf. Proposition 4.2).

Remark 8.2. In fact [CK] contains only classification of finite irreducible representations of Vir. The classification of all rank one Vir-modules is obtained by using 
Remark 8.1 or by the following simple argument: if $V=\mathcal{A} v$ is a free of rank one nontrivial representation of $V i r$, then it contains a minimal rank one submodule $W$ (see Corollary 8.1 below), which is clearly irreduciblt. Let $w=p(\partial) v$ be a generator for $W$; we can assume $p$ to be a monic polynomial. Then by classification of irreducibles of $V i r$, we have:

$$
p(\partial+\lambda) L_{\lambda} v=L_{\lambda} w=(c+\partial+\Delta \lambda) w=(c+\partial+\Delta \lambda) p(\partial) v
$$

If $L_{\lambda} v=q(\partial, \lambda) v$, then clearly $p(\partial+\lambda)=(c+\partial+\Delta \lambda)$ and $q(\partial, \lambda)=p(\partial)$, showing $\Delta=1$ and $L_{\lambda} v=(c+\partial) v$.

What we want to investigate now is how basic results from Lie algebra representation theory extend to the conformal algebra case. First, let us establish a few facts:

Lemma 8.2. If $R$ is a conformal algebra and $V$ is an $R$-module, then $R$ acts trivially on $\operatorname{Tor} V$.

Proof. $R$ acts via conformal linear maps. Use Proposition 3.2.

Corollary 8.1. Let $V_{0}$ be the intersection of all submodules of the $R$-module $V$ having the same rank as $V$. Then $R \cdot V \subset V_{0}$.

Lemma 8.3. If $R$ is a conformal algebra and $V$ its representation, then Tor $R$ acts trivially on $V$.

Proof. Tor $R$ acts via elements of Tor $(g c V)$, which is is zero (Remark 3.1).

Definition 8.2. Let $R \ni r \mapsto \phi_{r}(\lambda) \in \mathbb{C}[\lambda]$ be a $\mathbb{C}$-linear map. A $\phi$-weight space of a module $V$ over a conformal algebra $R$ is the $R$-invariant subspace

$$
V_{\phi}=\left\{v \in V \mid a_{\lambda} v=\phi_{a}(\lambda) v, a \in R\right\}
$$

A non-zero vector $v$ from $V_{\phi}$ is called a weight vector with weight $\phi$. 
Remark 8.3. If $V_{\phi} \neq 0$ (in which case $\phi$ is called a weight of $V$ ), then $\phi_{\partial a}(\lambda)=$ $-\lambda \phi_{a}(\lambda)$.

Theorem 8.4. (Conformal version of Lie's Theorem) Let $R$ be a finite rank solvable conformal algebra. Then any non-trivial irreducible finite rank conformal representation $V$ of $R$ is free of rank one: $V=\mathcal{A} v$, where $v$ is a weight vector.

In order to prove this statement, we need a simple lemma about finite solvable conformal algebras:

Lemma 8.4. If $R$ is a finite solvable conformal algebra, then the rank of $R^{\prime}$ is strictly lower than that of $R$.

This is an easy corollary of the following proposition.

Proposition 8.2. If $R$ is a finite conformal algebra such that $r k R^{\prime}=r k R$ then $R^{(n)}=R^{\prime}$ for all $n \geq 1$.

Proof. If $\operatorname{rk} R^{\prime}=\operatorname{rk} R$, then $R_{(n)} R \subset P(\partial) R$ for some non-zero polynomial $P \in \mathcal{A}$ and all $n$. Hence the second derived algebra $R^{(2)}=\left[R^{\prime} \cdot R^{\prime}\right]$ contains $\left\langle P(-\partial) P(\partial+\lambda)\left[R_{\lambda} R\right]\right\rangle$ which is equal to $\left\langle\left[R_{\lambda} R\right]\right\rangle$ by Lemma 3.1 , hence to $R^{\prime}$.

Proof of Theorem 8.4. Since Tor $R$ always acts trivially (Lemma 8.3), we can assume $R$ to be free as an $\mathcal{A}$-module. We prove the statement by induction on the rank of $R$. We have

$$
R \supset R^{(1)} \supset R^{(2)} \supset \ldots \supset R^{(n+1)}=0
$$

with $R^{(n)}$ non-zero and commutative. Then $R$ acts on $R^{(n)}$ via the adjoint representation, $R^{(n)}$ acting trivially. So we get a representation of $R / R^{(n)}$ in $R^{(n)}$, hence by inductive assumption (cf. Lemma 8.4) we have a weight vector $b \in R^{(n)}$ with weight independent of $\partial$ (if $R$ itself is commutative, any vector is a weight vector of weight zero for this action). This means $\left[a_{\lambda} b\right]=f_{a}(\lambda) b$ for all $a \in R$. Denote by $\mathfrak{b}_{i}$ the

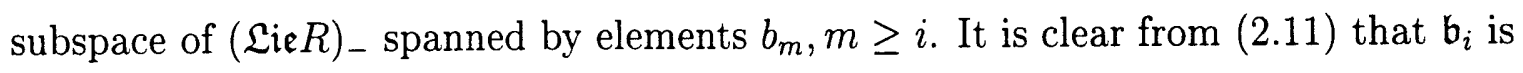
normalized by $(\mathfrak{L i e} R)_{-}$. But $\mathfrak{b}_{\imath}$ kills a vector $u \in V$ for $i$ sufficiently large, by local 
nilpotency assumption (8.1). Let $V_{\jmath}$ be the set of all vectors killed by $\mathfrak{b}_{\jmath}$ and let $U$ be $V_{N}$ for $N$ minimal such that $V_{N} \neq(0)$. Because of Lemma 8.1, when $N>0, U$ is a vector space of finite dimension, and it is invariant under the action of $(\mathfrak{L i e} R)_{-}$, since $\mathfrak{b}_{N}$ is normalized by $(\mathfrak{L} \mathfrak{i} \mathfrak{e} R)_{-}$. But now we can consider the image of $(\mathfrak{L} \mathfrak{i} \mathfrak{e} R)_{-}$ inside $\mathfrak{g l} U$. This will be a solvable Lie algebra, hence we have an eigenvector $v$ by the classical Lie's theorem (see e.g. [S]), hence $\dot{V}=\mathcal{A} v$ and theorem is proved.

In the other case $N=0, b$ kills some non-zero vector $v: b_{\lambda} v=0$. Since $\mathcal{A} b$ forms an ideal, the set of vectors killed by $b$ is a non-zero submodule of $V$, hence $V$ itself. Hence $R / \mathcal{A} b$ acts on $V$. But $R / \mathcal{A} b$ is of lower rank than $R$, it is still solvable, and $V$ is its irreducible representation. Then theorem follows by induction.

Corollary 8.2. The derived algebra of a solvable conformal algebra $R$ always acts trivially on an irreducible representation of $R$.

Lemma 8.5. If $V=\mathcal{A} v$ is a free of rank one representation of a solvable conformal algebra $R$, then $V$ is either trivial or irreducible.

Proof. It follows form Remark 8.1. We also give here an alternative proof. If $V$ is non-trivial, there is a minimal submodule $W$ of $V$ of rank one (Corollary 8.1). If $W \neq V$, then $W$ is spanned by a vector $w=p(\partial) v$, with $p$ non-constant. The module $W$ is irreducible, hence it is a weight module. We have:

$$
p(\partial+\lambda) q(\partial, \lambda) v=p(\partial+\lambda) s_{\lambda} v=s_{\lambda} w=\phi_{s}(\lambda) w=\phi_{s}(\lambda) p(\partial) v
$$

for some polynomial $q(\partial, \lambda)$. This gives $\phi_{s}(\lambda) p(\partial)=p(\partial+\lambda) q(\partial, \lambda)$. Since the polynomial $p$ is non-constant, it must have a complex zero $c$. Substituting $c-\lambda$ for $\partial$, we get $\phi_{s}(\lambda) p(c-\lambda)=0$, hence $\phi_{s}(\lambda)=0$. But this tells us that the action of $S$ on $W$ is trivial, giving a contradiction. Hence $W=V$, and $V$ is irreducible.

Corollary 8.3. Let $R$ be a finite solvable conformal algebra and $V$ a finite conformal module for $R$. Then it is always possible to find a family of submodules

$$
\operatorname{Tor} V=V_{0} \subset V_{1} \subset \ldots \subset V_{N}=V
$$


such that $V_{i+1} / V_{i}$ is free of rank one for $i \geq 0$. The action of $R$ on $V_{i+1} / V_{\imath}$ is either trivial or as in Theorem 8.4. In particular, $R^{\prime}$ is a nilpotent conformal algebra.

If $V$ is a free $\mathcal{A}$-module, then we can choose an $\mathcal{A}$-basis of $V$ in which the action of $R$ is expressed via upper triangular matrices.

Corollary 8.3 implies:

Theorem 8.5. If $R$ is a finite solvable conformal algebra, then there is a sequence of ideals

$$
\text { Tor } R=R_{0} \subset R_{1} \subset \ldots \subset R_{N}=R
$$

such that $R_{\imath+1} / R_{\imath}$ is free of rank one as an $\mathcal{A}$-module. In particular, if $R$ is free as an $\mathcal{A}$-module, it can be obtained by a sequence of extensions by commutative conformal algebras that are free as $\mathcal{A}$-modules.

We finish by proving the useful analogue of a classical result of Lie representation theory (see e.g. $[\mathrm{S}]$ ) sometimes referred to as the Cartan-Jacobson theorem.

Theorem 8.6. (Conformal version of Cartan-Jacobson Theorem) Let $R$ be a finite conformal algebra which has a finite irreducible faithful representation $V$. Then $R$ is isomorphic to one of the following conformal algebras:

(i) Cur $\mathfrak{g}$, where $\mathfrak{g}$ is a non-zero reductive Lie algebra whose center is at most one-dimensional;

(ii) the standard semi-direct sum of Vir and Cur $\mathfrak{g}$, where $\mathfrak{g}$ is as in (i) or zero.

This result allows us to easily prove the conformal version of Engel's theorem for Lie algebras.

Corollary 8.4. Let $R \subset g c V$ be a finite conformal algebra, with $V$ of finite rank. If $a_{n}$ is nilpotent on $V$ for any $a \in R$ and $n \in \mathbb{Z}_{+}$and $V \neq 0$, then there exists $a$ non-zero $v \in V$ such that $a_{\lambda} v=0$ for all $a \in R$.

Proof. By contradiction: suppose there is no vector killed by the whole of $R$. First of all, $V$ must be torsionless, due to Proposition 3.2. 
Let us take a maximal proper submodule $W$ (there always exists one). If $W=0$ then $V$ is irreducible. But Theorems 8.1-8.3 and 8.6 classify all faithful irreducible representations of conformal algebras, and no one of them is acted on by all nilpotent maps. This means that all of $R$ acts as 0 , If $W \neq 0$ and $\mathrm{rk} W<\operatorname{rk} V$ then the statement holds for $W$ by induction on $\operatorname{rk} V$.

Let us take the intersection $W_{0}$ of all submodules of $V$ with the same rank as $V$. By Corollary 8.1, $R \cdot V \subset W_{0}$.

If $\mathrm{rk} W_{0}=\mathrm{rk} V$, then $W_{0}$ has no submodules of the same rank as $V$, so we can use the above proof for $W_{0}$. If $0<\mathrm{rk} W_{0}<\mathrm{rk} V$ then we have found a submodule of lower rank than $V$, and we can use induction. If $W_{0}=0$ then $R \cdot V=0$.

Corollary 8.5. (Conformal version of Engel's Theorem) If $R$ is a finite rank conformal algebra and all elements of $R$ are ad-nilpotent then $R$ is a nilpotent conformal algebra.

Proof. By induction on $\mathrm{rk} R$. Since ad $R \subset g c R$ satisfies hypotheses of Corollary 8.4, there is $x \neq 0$ in $R$ such that $R \cdot x=0$, i.e. the center $Z$ of $R$ is non-trivial. But $R / Z$ also consists of ad-nilpotent elements and its rank is less than that of $R$, since $g c R$ is free, and the rank of $Z \subset g c R$ is therefore of rank at least one.

Inductive hypothesis shows that $R / Z$ is nilpotent. But it is clear that $R$ is nilpotent if and only if $R / Z$ is.

We will divide the proof of Theorem 8.6 in several lemmas.

Lemma 8.6. If $R$ is a finite conformal algebra having a faithful irreducible finite representation $V$ then the radical of $R$ is a commutative ideal.

Proof. Let $J=\operatorname{Rad} R$. Since $J$ is solvable, there is a $v \in V$ that is a common eigenvector for $J$; furthermore, we know that $J$ acts on $v$ with weights that are independent of $\partial$ (Theorem 8.4). Hence $J^{\prime}=[J \cdot J]$ is an ideal that kills $v$. But the set of all vectors killed by $J^{\prime}$ is a non-zero submodule, so it is the whole $V$. By faithfulness of $V, J^{\prime}=0$. 
Definition 8.3. Let $J$ be a conformal algebra and let $V$ be a $J$-module. Let $J \ni a \mapsto \phi_{a}(\lambda) \in \mathbb{C}[\lambda]$ be a linear map. For a non-negative integer $n$ denote by $V_{\phi}^{n}$ the subspace of all elements of $V$ that are killed by $n$ factors of the form $a_{\lambda}-\phi_{a}(\lambda)$, where $a \in J, \lambda \in \mathbb{C}$. The generalized weight space of $V$ corresponding to $\phi$ is

$$
V_{\phi}=\bigcup_{n=1}^{\infty} V_{\phi}^{n}
$$

(Note that $V_{\phi}^{1}$ is the ordinary weight space).

Lemma 8.7. Let $V$ be an $R$-module, and let $J$ be an ideal of $R$ and $V_{\phi}=\cup_{n \geq 1} V_{\phi}^{n}$ be a generalized weight space for $J$. Then for each $n \in \mathbb{Z}_{+}$we have:

(a) $\partial V_{\phi}^{n} \subset V_{\phi}^{n+1}$.

(b) Each $V_{\phi}^{n}$ is $J$-invariant.

(c) $R \cdot V_{\phi}^{n} \subset V_{\phi}^{n+1}$.

(d) $V_{\phi}^{n+1}=\left\{v \in V \mid\left(a_{\lambda}-\phi_{a}(\lambda)\right) v \in V_{\phi}^{n}\right.$ for all $\left.a \in J, \lambda \in \mathbb{C}\right\}$.

(e) $V_{\phi}$ is an $R$-submodule of $V$.

Proof. (d) is clear. (a) is proved by induction, observing that $\left(a_{\lambda}-\phi_{a}(\lambda)\right)(\partial v)=$ $\partial\left(\left(a_{\lambda}-\phi_{a}(\lambda)\right) v\right)+\lambda a_{\lambda} v ;(\mathrm{d})$ takes care of the basis of induction, since if $v \in V_{\phi}^{1}$, then $\left(a_{\lambda}-\phi_{a}(\lambda)\right)(\partial v)=\lambda a_{\lambda} v \in V_{\phi}^{1}$. (b) is immediate since $a_{\lambda} v=\left(a_{\lambda}-\phi_{a}(\lambda)\right) v+\phi_{a}(\lambda) v$.

Proof of (c) is as follows: If $v \in V_{\phi}^{n}$ then for all $r \in R, a \in J$,

$$
r_{\lambda} a_{\mu} v-a_{\mu} r_{\lambda} v=\left[r_{\lambda} a\right]_{\lambda+\mu} v
$$

From this we get $r_{\lambda}\left(a_{\mu}-\phi_{a}(\mu)\right) v-\left(a_{\mu}-\phi_{a}(\mu)\right) r_{\lambda} v=-\left[r_{\lambda} a\right]_{\lambda+\mu} v$ hence, since $\left[r_{\lambda} a\right] \in J$ and $\left(a_{\mu}-\phi_{a}(\mu)\right) v \in V_{\phi}^{n-1},\left(a_{\mu}-\phi_{a}(\mu)\right) r_{\lambda} v \in V_{\phi}^{n}$ by inductive hypothesis and (a). (e) is implied by (a) and (c).

It will be useful below to set an appropriate basis in order to compare matrix representations of the action of two elements that need to be equal. 
Lemma 8.8. There exists an $\mathcal{A}$-basis $\left\{v_{1}^{1}, v_{2}^{1}, \ldots, v_{k_{1}}^{1}, v_{1}^{2}, \ldots, v_{k_{2}}^{2}, v_{1}^{3}, \ldots\right\}$ of $V_{\phi}$ such that

$$
V_{\phi}^{n}=\left\{\sum_{i, \jmath} p_{\jmath}^{\imath}(\partial) v_{\jmath}^{\imath} \mid \operatorname{deg} p_{\jmath}^{\imath}(\partial) \leq n-i\right\}
$$

Proof. For every $i \in \mathbb{Z}_{+}$let $\left\{v_{\jmath}^{2}\right\}_{\jmath \in J_{2}}$ be a $\mathbb{C}$-basis of $V_{\phi}^{i}$ modulo $V_{\phi}^{i} \cap \mathcal{A} V_{\phi}^{i-1}$. We want to show that all $\left\{v_{j}^{\imath}\right\}$ are linearly independent over $\mathcal{A}$. In particular, if $V_{\phi}$ is finite, then $\left\{v_{j}^{2}\right\}$ is a finite set of vectors.

If we have a non-trivial relation between the $v_{\jmath}^{i}$ s, we can choose

$$
\sum_{\substack{i=1 ., N^{N-1} \\ j}} p_{\jmath}^{i}(\partial) v_{\jmath}^{i}+\sum_{\jmath} q_{\jmath}(\partial) v_{\jmath}^{N}=0
$$

with $q_{\jmath} \neq 0$ for some $j$, and of minimal maximal degree $d$ of the $q_{j}$ 's. Then $d=0$. In fact, if $d>0$, then applying $a_{\lambda}-\phi(\lambda)$ to both sides of (8.4), one gets:

$$
\sum_{\substack{\imath=1 . N-1 \\ \jmath}} \tilde{p}_{\jmath}^{\imath}(\partial, \lambda) v_{\jmath}^{\imath}+\phi_{a}(\lambda) \sum_{\jmath}\left(q_{j}(\partial+\lambda)-q_{\jmath}(\partial)\right) v_{j}^{N}=0
$$

for some polynomials $\tilde{p}_{j}^{2}$. If the degree of $\phi_{a}$ is $m$, the coefficient of $\lambda^{m+d}$ in (8.5) gives a linear relation as in (8.4) where all $q_{j}$ 's are constant, and not all of them are 0.

Once we know $d=0,(8.4)$ shows that $v=\sum_{j} q_{j} v_{j}^{N}$ is a non-trivial $\mathbb{C}$-linear combination of the $v_{j}^{N}$ which lies in $\mathcal{A} V_{\phi}^{N-1}$ by construction. But $v$ represents a non-zero class in $V_{\phi}^{N} /\left(V_{\phi}^{N} \cap \mathcal{A} V_{\phi}^{N-1}\right)$, giving a contradiction. Therefore, the $v_{\jmath}^{\imath}$ 's are linearly independent, and they clearly span $V_{\phi}$.

We are left with showing that $V_{\phi}^{n}=\left\{\sum_{i, j} p_{\jmath}^{i}(\partial) v_{j}^{i} \mid \operatorname{deg} p_{\jmath}^{i} \leq n-i\right\}$. This is obviously true for $n=1$, and we want to establish it for all $n$ using induction on $n$. Every element inside $V_{\phi}^{n+1}$ is clearly an $\mathcal{A}$-linear combination of the $v_{j}^{i}, i \leq n+1$. Let

$$
\sum_{i=1} p_{j}^{i}(\partial) v_{j}^{i}+\sum_{j} q_{j}(\partial) v_{\jmath}^{N}
$$

be an element of $V_{\phi}^{n+1}$. We show by induction on $N$ that polynomials $p_{j}^{i}$ and $q_{j}$ are 
as desired. Applying $a_{\lambda}-\phi(\lambda), \lambda \in \mathbb{C}$, one gets

$$
\sum_{\substack{\imath=1, \ldots N-1 \\ j}} \tilde{p}_{j}^{2}(\partial, \lambda) v_{\jmath}^{i}+\phi_{a}(\lambda) \sum_{j}\left(q_{\jmath}(\partial+\lambda)-q_{\jmath}(\partial)\right) v_{\jmath}^{N}
$$

for some choice of polynomials $\tilde{p}_{j}^{2}$, and this must lie in $V_{\phi}^{n}$. But inductive assumption tells us that the degree in $\partial$ of $q_{\jmath}(\partial+\lambda)-q_{\jmath}(\partial)$ must be $\leq n-N$ for all $\lambda \in \mathbb{C}$, hence $\operatorname{deg} q_{j} \leq n+1-N$.

If so, the $\mathcal{A}$-linear combination $\sum_{j} q_{\jmath}(\partial) v_{j}^{N}$ certainly belongs to $V_{\phi}^{n+1}$, hence we are left with proving the statement for

$$
\sum_{\substack{\imath=1 . N-1 \\ j}} p_{\jmath}^{\imath}(\partial) v_{\jmath}^{\imath}
$$

which is true by inductive assumption.

We will refer to any basis of $V_{\phi}$ obtained in this way as to a standard basis. Matrix representations of conformal linear maps are analogous to those of linear maps between vector spaces. If $A(\partial, \lambda)$ is the matrix representing the action of $a_{\lambda}$ and $B(\partial, \mu)$ is that representing the action of $b_{\mu}$, then the matrix representing $a_{\lambda} b_{\mu}$ is clearly given by the product matrix $A(\partial, \lambda) B(\partial+\lambda, \mu)$.

We are going to consider generalized weight spaces for the action of $J=\operatorname{Rad} R$ on $V$. They come useful, since they are fixed by the action of $R$. Since $J$ is solvable, we know by the conformal version of Lie's theorem (Theorem 8.4) that at least one of the weight-spaces is non-trivial. Let $\phi$ be the corresponding weight. Note that $\phi \neq 0$, since otherwise $V_{\phi}^{1}$ is $R$-invariant, hence $V=\mathcal{A} V_{\phi}^{1}, J$ acts trivially on $V$, and Theorem 8.6 follows from Proposition 8.1.

From now on, $R$ will be a finite conformal algebra, $J$ its radical, $V$ a faithful irreducible finite $R$-module such that $V=V_{\phi}$, the generalized weight space with respect to the action of $J$ for the weight $\phi \neq 0$, for which $V_{\phi}^{1} \neq 0$.

Lemma 8.9. The rank of $J$ is at most one.

Proof. Suppose that $\mathrm{rk} J>1$. Then, by Remark 8.3, $\phi_{\partial a}=-\lambda \phi_{a}$, hence we can 
always find a non-zero $a \in J$ for which $\phi_{a}=0$. Now, all elements in $J$ whose weight is 0 form an ideal $J_{0}$ of $R$. In fact, since the action of $r \in R$ increases the filtration by one (Lemma 8.7c), and $a_{\lambda}-\phi_{a}(\lambda), \lambda \in \mathbb{C}$, obviously decreases it by one, their matrix representation in any fixed standard basis is given by the following block matrices for the filtration $\left\{V_{\phi}^{n}\right\}$ :

$$
\begin{aligned}
& r_{\lambda}=\left(\begin{array}{cccccc}
* & . & . & . & \ddots & . \\
R_{1} & * & . & . & . & \\
0 & R_{2} & * & . & . \\
\vdots & \ddots & \ddots & \ddots & \\
0 & \ldots & 0 & R_{n-1} & *
\end{array}\right)
\end{aligned}
$$

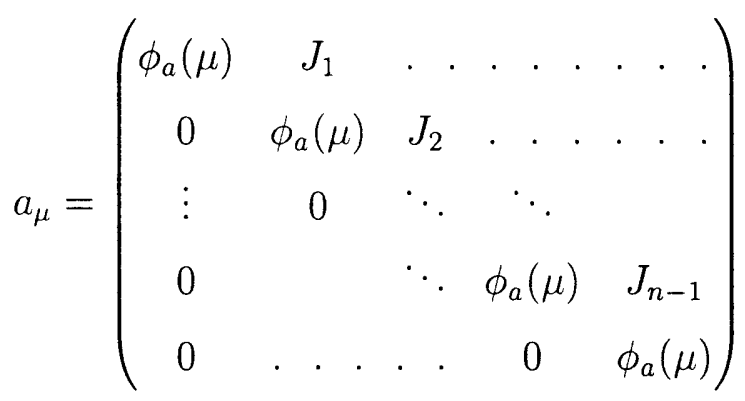

where $R_{\imath}=R_{\imath}(\lambda)$ and $J_{\imath}=J_{\imath}(\mu)$ are independent of $\partial$ (cf. Lemma 8.8); then taking trace of the matrices representing both sides of

$$
r_{\lambda} a_{\mu}-a_{\mu} r_{\lambda}=\left[r_{\lambda} a\right]_{\lambda+\mu}
$$

we easily get that if $\phi_{a}$ is zero, then $\phi_{\left[r_{\lambda} a\right]}$ is also zero. The same reasoning as before shows that $J_{0}$ acts trivially on $V$, a contraiction with faithfulness of the $R$-module $V$.

Once we know $J$ is commutative of rank one, we can consider the adjoint representation of $R / J$ on $J$. This is a rank one representation of a semi-simple conformal algebra. We know from Theorem 8.1 that the current part of $R / J$ must act as 0 , and that of all the Virasoro algebras, only one can act non-trivially. Let $S \subset R$ be the centralizer of $J$ in $R$. 
Lemma 8.10. S stabilizes the filtration $\left\{V_{\phi}^{n}\right\}$.

Proof. By definition of $S,[S \cdot J]=0$. Then using this in (8.2) gives the proof.

This tells us that in the standard basis the matrices representing the action of elements from $S$ are block upper triangular for the filtration $\left\{V_{\phi}^{n}\right\}$, with diagonal blocks independent of $\partial$. In particular, if $S / J$ contains Vir's, diagonal blocks in their matrix representation are trivial (by Theorem 8.2). Hence their action is trivial on $V$, so $S / J$ is a current conformal algebra by Theorem 7.1 .

Lemma 8.11. All central extensions of a finite simple conformal algebra $R$ by a free $\mathcal{A}$-module of rank one are trivial.

Proof. Let $R$ be a central extension of a finite simple current conformal algebra $\operatorname{Cur} \mathfrak{g}$ by a rank one center $\mathcal{A}$. The $\lambda$-bracket in such a conformal algebra is given by

$$
\left[g_{\lambda} h\right]=[g, h]+\alpha_{\lambda}(g, h),
$$

where $g, h \in \mathfrak{g} \subset$ Cur $\mathfrak{g}$ and $\alpha_{\lambda}(g, h) \in \mathcal{A}[\lambda]$. Then axiom (C4) gives

$$
\alpha_{\lambda}(a,[b, c])-\alpha_{\mu}(b,[a, c])=\alpha_{\lambda+\mu}([a, b], c)
$$

and setting $\alpha_{\lambda}(g, h)=\sum_{i} \lambda^{2} \alpha_{\imath}(g, h)$ :

$$
\sum_{i} \lambda^{i} \alpha_{i}(a,[b, c])-\sum_{\jmath} \mu^{\jmath} \alpha_{\jmath}(b,[a, c])=\sum_{k}(\lambda+\mu)^{k} \alpha_{k}([a, b], c),
$$

for every $a, b, c \in \mathfrak{g}$.

This immediately shows $\alpha_{i}=0$ for $i \geq 2$, since $\mathfrak{g}^{\prime}=\mathfrak{g}$. Moreover:

$$
\begin{gathered}
\alpha_{0}(a,[b, c])-\alpha_{0}(b,[a, c])=\alpha_{0}([a, b], c), \\
\alpha_{1}(a,[b, c])=\alpha_{1}([a, b], c) .
\end{gathered}
$$


Axiom (C3) is equivalent to

$$
\alpha_{0}(a, b)+\alpha_{0}(b, a)=\partial \alpha_{1}(a, b)
$$

It follows from (8.9) and (8.10) that $\alpha_{1}$ is a symmetric invariant $\mathcal{A}$-valued bilinear form on $\mathfrak{g}$, hence it is a multiple of the Killing form $(\cdot \mid \cdot): \alpha_{1}(g, h)=p(\partial)(g \mid h)$.

Set $\beta(g, h)=\alpha_{0}(g, h)-\frac{1}{2} \partial p(\partial)(g \mid h)$. Then (8.10) implies skew-symmetry of $\beta$ and (8.8) translates as

$$
\beta([a, b], c)-\beta(a,[b, c])+\beta(b,[a, c])=\frac{1}{2} \partial p(\partial)([a, b] \mid c) .
$$

Plugging in $a=h \in \mathfrak{h}, b=e_{\alpha}, c=e_{-\alpha}$ for some root $\alpha$, we get:

$$
\beta\left(h,\left[e_{\alpha}, e_{-\alpha}\right]\right)=\frac{1}{2} \partial p(\partial) \alpha(h)\left(e_{\alpha} \mid e_{-\alpha}\right) .
$$

If $h_{\alpha}$ is the bracket of root vectors $e_{\alpha}, e_{-\alpha}$ such that $\left(e_{\alpha} \mid e_{-\alpha}\right)=1$, we get $\beta\left(h_{\alpha}, h_{\alpha}\right)=\frac{1}{2} \partial p(\partial) \alpha\left(h_{\alpha}\right)$. But the left-hand side of (8.11) must be 0 due to skewsymmetry of $\beta$. Hence $p=0$. This shows that $\alpha_{1}$ is 0 on the whole $\mathfrak{g}$, hence $\alpha_{0}$ is a Lie algebra 2-cocycle for $\mathfrak{g}$, so it is trivial, since any central extension of the simple Lie algebra $\mathfrak{g}$ is trivial.

In the case of a central extension of $\operatorname{Vir}$, axiom (C4) for the $\lambda$-bracket

$$
\left[L_{\lambda} L\right]=(\partial+2 \lambda) L+p(\partial, \lambda)
$$

gives the following condition on $p$ :

$$
(\partial+\lambda+2 \mu) p(\partial, \lambda)-(\partial+2 \lambda+\mu) p(\partial, \mu)=(\lambda-\mu) p(\partial, \lambda+\mu)
$$

Setting $\mu=0$ in (8.12) we get $\partial p(\partial, \lambda)=(\partial+2 \lambda) p(\partial, 0)$, whence $p(\partial, \lambda)=q(\partial)(\partial+$ $2 \lambda)$, for $q(\partial)=\frac{p(\partial)}{\partial}$. Then $L+q(\partial)$ is a standard generator of a Virasoro conformal algebra. 
Lemma 8.12. If $R$ is a finite semi-simple conformal algebra, then all central extensions of $R$ by a free $\mathcal{A}$-module of rank one are trivial.

Proof. As an $\mathcal{A}$-module, a semi-simple conformal algebra $R$ is a finite direct sum $\oplus_{\imath} S_{i}$ of subalgebras $S_{\imath}$ (Theorem 7.1). We can make a 2-cocycle $\alpha_{\lambda}(\cdot, \cdot)$ on $R$ trivial on $S_{\imath} \times S_{i}$ for all $i$. We will call $\left.\alpha_{\lambda}\right|_{S_{\imath} \times S_{\jmath}}$ a cross-extensions of $S_{i}$ and $S_{\jmath}$. Our goal is to prove that cross-extensions of a 2-cocycle on $R$ are zero if its restrictions on all $S_{i}$ are zero. Without loss of generality, we can assume $R$ to be the (semi-direct) sum of two simple algebras.

If $R=C u r \mathfrak{g}$ is a semi-simple current conformal algebra, one shows as in Lemma 8.11 that $\alpha_{1}$ is an invariant bilinear form on $\mathfrak{g}$, hence $\alpha_{1}(g, h)=0$ if $g$ and $h$ belong to distinct simple ideals. So $\alpha_{1}$ is identically zero on $\mathfrak{g}$, hence $\alpha_{0}$ is a Lie algebra 2-cocycle on $\mathfrak{g}$, hence it is trivial (since $\mathfrak{g}$ is a semi-simple Lie algebra). This takes care of cross extensions of a simple conformal algebra by another simple conformal algebra.

Next, we need to figure out what $\alpha_{\lambda}(L, g)$ can be if $L$ is the standard generator of $V i r$, and $g \in \operatorname{Cur} \mathfrak{g}$, with $\mathfrak{g}$ a finite-dimensional simple Lie algebra. In a semi-direct sum, $L$ will act trivially or in the standard way on $g$. Then setting $a=L, b=g$, $c=h$, with $g, h \in \mathfrak{g}$, in (8.7) gives $\alpha_{\lambda}(L,[g, h])=0$ as soon as $\alpha_{\lambda}$ is zero on $\mathfrak{g} \times \mathfrak{g}$. If we make $\alpha_{\lambda}$ zero in advance, this shows that $\alpha_{\lambda}(L, g)=0$ if $g$ lies inside the derived Lie algebra $\mathfrak{g}^{\prime}$ which equals $\mathfrak{g}$ when $\mathfrak{g}$ is simple.

In order to show that also cross-extensions of Vir by Vir are zero, it is enough to substitute $a=L^{1}, b=c=L^{2}$ in (8.7), where $L^{1}$ and $L^{2}$ are standard generators for the two Virasoro conformal algebras.

Remark 8.4. (a) The universal central extension of the conformal algebra Vir has one-dimensional (over $\mathbb{C}$ ) center and the corresponding cocycle is a multiple of $\alpha_{\lambda}(L, L)=\lambda^{3}$ (of course the associated formal distribution Lie algebra is the usual Virasoro algebra).

(b) The center of the universal central extension of $C u r \mathfrak{g}$, where $\mathfrak{g}$ is a finitedimensional semi-simple Lie algebra is canonically identified with the space $B$ of all 
invariant bilinear forms on $\mathfrak{g}$ and the corresponding cocycle is $\alpha_{\lambda}^{f}(a, b)=f(a, b)$, $f \in B$, where $a, b \in \mathfrak{g}$ (of course, for simple $\mathfrak{g}$, the associated formal distribution Lie algebra is the usual affine Kac-Moody algebra).

(c) For an arbitrary semi-simple conformal algebra the universal central extension is the obvious combination of (a) and (b).

Corollary 8.6. $S=S / J \oplus J$ (direct sum of conformal algebras).

If $R=S$, i.e. $J$ is centralized by the whole $R$, we are finished, since $S$ is the current conformal algebra associated to a reductive Lie algebra with one-dimensional center. If $R \neq S$, we need to do some extra work.

We are in the following situation: $R$ is an extension of $R / J$ by $J$ which is trivial on $S / J$, and $R / S=V i r$. From now on, denote by $a$ a generator of $J$, by $\phi$ the polynomial $\phi_{a}$, and by $L$ a representative in $R$ of the standard generator for the quotient conformal algebra $R / S$. It is clear that we can choose $L$ to act on $S / J$ in the standard way, and with no contribution in $J$ (since $S / J$ is isomorphic to $S^{\prime}$ ). Next, we prove:

Lemma 8.13. $\left[L_{\lambda} a\right]=(\partial+\lambda) a$.

Proof. By now we only know that $\left[L_{\lambda} a\right]=p(\partial, \lambda) a$ for some polynomial $p \in \mathbb{C}[\partial, \lambda]$. Writing down matrix representations of the action of $L$ and $a$ in the standard basis

$$
L_{\lambda}=\left(\begin{array}{ccccccc}
M_{1} & . & . & . & . & . & . \\
L_{1}(\lambda) & M_{2} & . & . & . & . & \\
0 & L_{2}(\lambda) & \ddots & & & \\
\vdots & \ddots & \ddots & M_{n-1} & \\
0 & \ldots & 0 & L_{n-1}(\lambda) & M_{n}
\end{array}\right)
$$




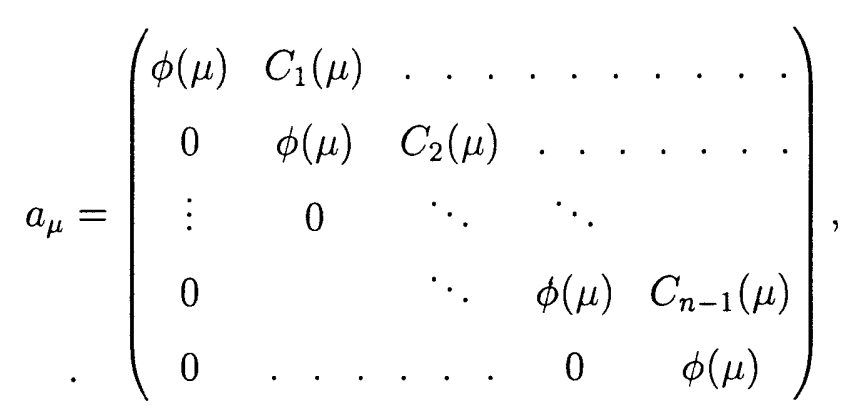

where $L_{i}(\lambda)$ 's and $C_{\imath}(\mu)$ 's are independent of $\partial$ and $M_{i}(\partial, \lambda)$ 's are linear in $\partial$ (cf. Lemma 8.8), and taking trace of matrices representing both sides of:

$$
\left[L_{\lambda}, a_{\mu}\right]=p(-\lambda-\mu, \lambda) a_{\lambda+\mu}
$$

we get

$$
\phi(\mu)\left(\sum_{i} \operatorname{Tr}\left(M_{\imath}(\partial, \lambda)-M_{\imath}(\partial+\mu, \lambda)\right)=p(-\lambda-\mu, \lambda) \phi(\lambda+\mu) \cdot \operatorname{rk}(V) .\right.
$$

Set $M(\partial, \lambda)=\operatorname{Tr} \sum_{\imath} M_{\imath}(\partial, \lambda)$ and write $M(\partial, \lambda)=M_{0}(\lambda)+N(\lambda) \partial$. Then (8.14) can be rewritten as

$$
-\mu \phi(\mu) N(\lambda)=p(-\lambda-\mu, \lambda) \phi(\lambda+\mu) \operatorname{rk}(V) .
$$

Setting $\mu=0$ in (8.15) we obtain $p(-\lambda, \lambda) \phi(\lambda)=0$ and since $\phi$ is not identically zero, $p(-\lambda, \lambda)=0$ for all $\lambda \in \mathbb{C}$. This means that $p(\partial, \lambda)=(\partial+\lambda) q(\partial, \lambda)$ for some other polynomial $q$. But the adjoint action of $L$ on $a$ defines a rank one representation of the Virasoro conformal algebra, and we know all such (Theorem 8.2). The only possible values of $q$ are zero and one. If $q$ is zero, then $L$ centralizes $J$ and $R=S$. If $q=1$ then $\left[L_{\lambda} a\right]=(\partial+\lambda) a$.

Remark 8.5. Substituting $p(\partial, \lambda)=\partial+\lambda$ in (8.15) it is immediate to show that $\phi$ must be a constant polynomial and $N(\lambda)=$ Id. Up to rescaling $a$ by a complex multiple, $\phi$ can therefore be made equal to 1 . 
The only $\lambda$-bracket we now need to take care of is $\left[L_{\lambda} L\right]$. In order to show it is nothing but $(\partial+2 \lambda) L$, or rather, that it can be made into this form up to shifting the representative $L$ by an element in $J$, we prove the following lemma.

Lemma 8.14. $L_{\lambda}-\partial$ stabilizes $V_{\phi}^{1}$.

Proof. Using Lemma 8.13 and axioms of an $R$-module, we get:

$$
\left[L_{\lambda}, a_{\mu}\right]=-\mu a_{\lambda+\mu}
$$

Applying both sides of this equation to $v \in V_{\phi}^{1}$, we get

$$
L_{\lambda} v-s_{\mu} L_{\lambda} v=-\mu v
$$

where $s_{\mu}$ is as in Proposition 6.2. Using the notation of Lemma 8.13, the left top block in the matrix representation of $-\mu a_{\lambda+\mu}$, obtained from (8.16), must be equal to $-\mu-C_{1}(\mu) L_{1}(\lambda)$. But by (8.17) this equals to $-\mu$, so $C_{1}(\mu) L_{1}(\lambda)=0$.

The way they have been obtained, it is clear that the columns of $C_{1}(\mu)$ are linearly independent over $\mathbb{C}$, otherwise we would get a linear combination of the $v_{\jmath}^{2}$ which lies in $V_{\phi}^{1}$. Therefore $L_{1}(\lambda)$ itself must be zero. This shows that $\mathcal{A} V_{\phi}^{1}$ is stabilized by the action of $L$ and that $L_{\lambda}-\partial$ acts independently of $\partial$ on a basis.

Combining Lemma 8.7b, Lemma 8.10 and Lemma 8.14, we see that $\mathcal{A} V_{\phi}^{1}$ is an $R$-submodule of $V$, hence $V=\mathcal{A} V_{\phi}^{1}$. So there is a basis of $V$ of eigenvectors for $a$, and the action of $a$ is given in this basis by the identity matrix.

Lemma 8.15. $L$ can be chosen such that $\left[L_{\lambda} L\right]=(\partial+2 \lambda) L$.

Proof. The matrix representation of the action of $L_{\lambda}$ in the eigenvector basis of $V$ is $\partial+C(\lambda)$, where $C$ is some matrix independent of $\partial$ (Lemma 8.14). Up to adding to $L$ an appropriate element $j$ from $J$, we can make the trace of $C(\lambda)$ equal to zero.

Let $\tilde{L}=L-j$. Then the action of $\left[\tilde{L}_{\lambda}, \tilde{L}_{\mu}\right]-(\lambda-\mu) \tilde{L}_{\lambda+\mu}$ is given by the matrix $\lambda C(\lambda)-\mu C(\mu)-(\lambda-\mu) C(\lambda+\mu)$, whose trace is clearly 0 . This shows that if 
$\left[\tilde{L}_{\lambda} \tilde{L}\right]=(\partial+2 \lambda) \tilde{L}+j^{\prime}, j^{\prime} \in J$, then the sum of elements on the diagonal of the matrix representation of $j^{\prime}$ is zero. Hence $j^{\prime}=0$, and $\tilde{L}$ is Virasoro-like.

End of proof of Theorem 8.6. By Lemma 8.9, $\mathrm{rk} J$ is at most one. If $J=0, R$ is semisimple. In view of Proposition 8.1, a finite semi-simple conformal algebra having a finite faithful irreducible representation is as in (i) or in (ii).

If $J \neq 0$, and $R$ centralizes $J$, then $R=R / J \oplus J$ is isomorphic to $C u r \mathfrak{g}$, where $\mathfrak{g}$ is a reductive Lie algebra with one-dimensional center.

If $J \neq 0$, and $R$ does not centralize $J$, then $R$ is the standard semi-direct sum of Vir with $S=S / J \oplus J$ which is the current conformal algebra associated to a reductive Lie algebra $\mathfrak{g}$ with one-dimensional center. 


\section{Chapter 9}

\section{A generalization: the case of several indeterminates}

The theory we have developed clearly dismisses most of the richness set forth by the Cartan Guillemin theorem. The most immediate way to include in our study higher rank Cartan type algebras (even though $K_{n}$ can never be realized as annihilation algebra of any $n$-conformal algebra) is by considering "local" families of formal distributions in several indeterminates. Proceeding like this we lose most of the physical motivation, but the algebraic objects we obtain still prove interesting. In this chapter I give a brief sketch of the theory of conformal algebras in $n$ indeterminates (or $n$-conformal algebras, as I call them) and try to approach the problem of listing all simple ones.

Let $V$ be a vector space. A $V$-valued formal distribution in $n$ variables $\phi$ is a formal power series

$$
\phi\left(z_{1}, z_{2}, \ldots, z_{n}\right)=\sum_{i_{1}, i_{2}, \ldots, i_{n} \in \mathbb{Z}} \phi_{i_{1}, i_{2}, \ldots, i_{n}} z_{1}^{-\imath_{1}-1} z_{2}^{-i_{2}-1} \ldots z_{n}^{-i_{n}-1} \in V\left[\left[z_{1}, z_{1}^{-1}, \ldots, z_{n}, z_{n}^{-1}\right]\right]
$$

We will often write $\phi(\boldsymbol{z})=\sum_{\boldsymbol{i} \in \mathbb{Z}^{n}} \phi_{\boldsymbol{i}} \boldsymbol{z}^{i-1}$, where $\boldsymbol{z}^{i}=z_{1}^{i_{1}} z_{2}^{i_{2}} \ldots z_{n}^{i_{n}}$ and $\mathbf{1}$ is the $n$-tuple $(1,1, \ldots, 1)$; elements $\phi_{i} \in V$ are called Fourier coefficients of $\phi$. If $\mathfrak{g}$ is a Lie algebra, two $\mathfrak{g}$-valued formal distributions $\phi, \psi$ in $n$ indeterminates are said "local" to each other if $(\boldsymbol{z}-\boldsymbol{w})^{\boldsymbol{N}}[\phi(\boldsymbol{z}), \psi(\boldsymbol{w})]=0$ for some $\boldsymbol{N} \in \mathbb{Z}^{n}$. 
As in the case of one indeterminate (Proposition 2.1) algebraic properties of local formal distributions in $n$ indeterminates are described by coefficient of the expansion:

$$
[a(\boldsymbol{z}), b(\boldsymbol{w})]=\sum_{\boldsymbol{i} \in \mathbb{Z}_{+}{ }^{n}} c^{i}(\boldsymbol{w}) \partial_{\boldsymbol{w}}^{(i)} \delta(\boldsymbol{z}-\boldsymbol{w})
$$

where $\delta(\boldsymbol{z}-\boldsymbol{w})=\delta\left(z_{1}-w_{1}\right) \delta\left(z_{2}-w_{2}\right) \ldots \delta\left(z_{n}-\dot{w}_{n}\right)$.

If we write $a_{(i)} b=c^{i}$ we get a family of bilinear products whose properties we can use as axioms of the algebraic structure we want to study:

Definition 9.1. $R$ is an $n$-conformal algebra if it is a $\mathbb{C}\left[\partial_{1}, \ldots, \partial_{n}\right]$-module endowed with a family of $\mathbb{C}$-bilinear products $(n), n \in \mathbb{Z}_{+}{ }^{n}$, satisfying the axioms:

(S1) $a_{(\boldsymbol{N})} b=0$ for $\boldsymbol{N}>>0$,

(S2) $\left(\partial_{\imath} a\right)_{(n)} b=-n_{i} a_{\left(n-e^{2}\right)} b, a_{(n)} \partial_{\imath} b=\partial_{i}\left(a_{(n)} b\right)+n_{i} a_{\left(n-e^{2}\right)} b$,

(S3) $a_{(\boldsymbol{n})} b=\sum_{\boldsymbol{j}}(\mathbf{- 1})^{\boldsymbol{n}+\boldsymbol{j}} \boldsymbol{\partial}^{(j)}\left(b_{(\boldsymbol{n}+\boldsymbol{j})} b\right.$,

(S4) $a_{(\boldsymbol{m})}\left(b_{(\boldsymbol{n})} c\right)-b_{(\boldsymbol{n})}\left(a_{(\boldsymbol{m})} c\right)=\sum_{\boldsymbol{j}=0}^{\boldsymbol{m}}\left(\begin{array}{c}\boldsymbol{m} \\ \boldsymbol{j}\end{array}\right)\left(a_{(\boldsymbol{j})} b\right)_{(\boldsymbol{m}+\boldsymbol{n}-\boldsymbol{j})} c$

where $\boldsymbol{e}^{i}$ is the $n$-tuple whose entries are all 0 but for the $i$-th one, which is 1 . If we write $\left[a_{\boldsymbol{\lambda}} b\right]=\sum_{\boldsymbol{n}} \boldsymbol{\lambda}^{(\boldsymbol{n})} a_{(\boldsymbol{n})} b,(\mathrm{~S} 0)$-(S3) are translated as follows:

(S0) $\left[a_{\boldsymbol{\lambda}} b\right]$ is polynomial in $\boldsymbol{\lambda}$,

$(\mathrm{S} 1)\left[\partial_{\imath} a_{\boldsymbol{\lambda}} b\right]=-\lambda_{i}\left[a_{\boldsymbol{\lambda}} b\right],\left[a_{\boldsymbol{\lambda}} \partial_{\imath} b\right]=\left(\partial_{\imath}+\lambda_{i}\right)\left[a_{\boldsymbol{\lambda}} b\right]$,

(S2) $\left[a_{\lambda} b\right]=-\left[b_{-\boldsymbol{\partial}-\boldsymbol{\lambda}} a\right]$,

(S3) $\left[a_{\boldsymbol{\lambda}}\left[b_{\boldsymbol{\mu}} c\right]\right]-\left[b_{\boldsymbol{\mu}}\left[a_{\boldsymbol{\lambda}} c\right]\right]=\left[\left[a_{\boldsymbol{\lambda}} b\right]_{\boldsymbol{\lambda}+\boldsymbol{\mu}} c\right]$.

We call $\left[a_{\lambda} b\right]$ the " $\lambda$-bracket" of $a$ and $b$ and completely describes the structure of $R$.

Remark 9.1. Notice that a 1-conformal algebra is just a conformal algebra, and that a 0 -conformal algebra is an ordinary Lie algebra.

Definition 9.2. If $V$ and $W$ are $\mathbb{C}\left[\partial_{1}, \ldots, \partial_{m}\right]$-modules, phi: $V \rightarrow W\left[\lambda_{1}, \ldots, \lambda_{n}\right]$ is an $n$-conformal linear map if $\phi_{\boldsymbol{\lambda}}\left(\partial_{\imath} v\right)=\left(\partial_{i}+\lambda_{i}\right) \phi_{\boldsymbol{\lambda}} v$. The space of all $n$-conformal linear maps from $V$ to $W$ is made into a $\mathbb{C}\left[\partial_{1}, \ldots, \partial_{m}\right]$ module by $\left(\partial_{i} \phi\right)_{\lambda} v=-\lambda_{i} \phi_{\lambda} v$, and is denoted by $\operatorname{Chom}(V, W)$. We will abuse the notiation by writing $\phi: V \rightarrow W$ as soon as it is clear that $\phi$ is $n$-conformal linear 
Remark 9.2. Elements in an $n$-conformal algebra $R$ act on $R$ via $n$-conformal maps. In particular, if ad $a: R \rightarrow R$ is such that $(\operatorname{ad} a)_{\boldsymbol{\lambda}} b=\left[a_{\boldsymbol{\lambda}} b\right]$, then ad $: R \rightarrow$ $\operatorname{Chom}(R, R)$ is a homomorphism of $\mathbb{C}\left[\partial_{1}, \ldots, \partial_{m}\right]$-modules. Setting the obvious $n$ conformal algebra structure on Cend $R=\operatorname{Chom}(R, R)$ (see Example 3.5) makes ad into a homomorphism of $n$-conformal algebras. Cend $R$ with this natural $\lambda$-bracket is denoted by $g c R$.

We will state a few facts about $n$-conformal algebras. We will not provide explicit proofs since they are clear generalizations of the ones given for ordinary 1-conformal algebras.

Proposition 9.1. Any $\phi \in \operatorname{Chom}(V, W)$ maps Tor $V$ to 0 . In particular, Tor $R$ is always a central ideal of the $n$-conformal algebra $R$.

Let the annihilation algebra $(\mathfrak{L i e} R)_{-}$be the image of $R\left[t_{1}, \ldots, t_{n}\right]$ inside $R\left[t_{1}, t_{1}^{-1}, \ldots, t_{n}, t_{n}^{-1}\right] /\left(\partial_{1}+\partial / \partial t_{1}, \ldots, \partial_{n}+\partial / \partial t_{n}\right)$ under the natural projection to the quotient. Let $a_{m}$ denote $a t^{m}=a t_{1}^{m_{1}} \ldots t_{n}^{m_{n}}$. Then the bracket

$$
\left[a_{m}, b_{n}\right]=\sum_{j}\left(\begin{array}{c}
m \\
j
\end{array}\right)\left(a_{(j)} b\right)_{m+n-j}
$$

is a well defined Lie bracket. Let $R$ be a finitely generated (as $\mathbb{C}\left[\partial_{1}, \ldots, \partial_{m}\right]$-module) $n$-conformal algebra. If $\left\{r^{\alpha}\right\}_{\alpha \in A}$ is a finite set of generators, we can define a filtration by subspaces and a topology on $(\mathfrak{L i e} R)_{-}$as in Proposition 4.2.

Then the Lie bracket 9.2 is continuous for this topology, and we can complete $(\mathfrak{L i e} R)_{\text {_ }}$ with respect to this filtration. The semi-direct product $\left\langle\partial_{1}, \ldots, \partial_{n}\right\rangle \ltimes\left(\widehat{\mathfrak{L i e} R)_{-}}\right.$ will then be a linearly compact Lie algebra, and will satisfy the conditions of Theorem 5.2. It is easy to show that for a semi-simple finitely generated (or finite) $n$-conformal algebra $R$ one can always find an ideal of $(\widehat{\mathcal{L i e} R)}$ - isomorphic to a nontrivial central extension of $S \hat{\otimes} \mathbb{C}\left[\left[t_{1}, \ldots, t_{r}\right]\right]$, where $S$ is as in Theorem 5.2. If $R$ is a simple conformal algebra, $(\widehat{\mathfrak{\mathcal { i } e} R)}$ - will then be of this kind. The classification of simple $n$-conformal algebra still requires a description of all possible actions of $\partial_{1}, \ldots, \partial_{n}$ on $\left(\widehat{\mathfrak{L i e} R)_{-}}\right.$, and a reconstruction theorem in the spirit of Proposition 5.1. The de- 
scription of all possible actions of $\partial_{1}, \ldots, \partial_{n}$ is given in [NW]. As far as Proposition 5.1 is concerned, we give here a very interesting generalization.

Let $R$ be a finite simple $n$-conformal algebra, and assume $R$ to be torsionless. If $\mathfrak{L}=(\mathfrak{L i e} R)_{-}$, denote the subspace of all power series $s\left(t_{1}, . ., t_{n}\right)$ in $\mathfrak{L}\left[\left[t_{1}, \ldots, t_{n}\right]\right]$ whose coefficients converge to 0 in the topology of $\mathfrak{L}$ and satisfying $\left(\partial_{\imath}+t_{i}\right) s\left(t_{1}, \ldots, t_{n}\right)=0$ by $\bar{R}$.

Claim: $\bar{R}$ is a $\mathbb{C}\left[\partial_{1}, \ldots, \partial_{m}\right]$-module. $R$ is naturally a subspace of $\bar{R}$ via the embedding $r \mapsto \sum_{i} r_{i} t^{i} . \bar{R}$ is finitely generated, and it is isomorphic to $R$ if $R$ is free. Furthermore there are no free submodules strictly between $R$ and $\bar{R}$.

We can now recover the conformal algebra structure on $\bar{R}$. Let $a=\sum_{i} a_{i} t^{i}, b=$ $\sum_{i} b_{i} t^{i}$. Define:

$$
c_{i}^{m}=\sum_{j}(-1)^{m+j}\left(\begin{array}{c}
m \\
j
\end{array}\right)\left[a_{j}, b_{m+i-j}\right] .
$$

Then $c^{m}=\sum c_{i}^{m} t^{i}$ lies in $\bar{R}$ too. Equation 4.9 shows that the element $c^{m}$ is "morally" the $j$-th product of $a$ and $b$. In this way we reconstruct a conformal algebra structure on $\bar{R}$ (it is not too difficult to show, using the filtration (4.6), that $c^{m}=0$ for $m>0$ ). Therefore $\bar{R}$ is a $n$-conformal algebra, and the embedding of $R$ in $\bar{R}$ is an injective homomorphism of $n$-conformal algebras.

Assuming $R$ was simple, $\bar{R}$ does not need to be so (for instance, it might contain $R$ itself as a proper ideal). However, $\bar{R}^{\prime}=[\bar{R} \cdot \bar{R}]$ is certainly simple (see Corollary 8.1 and Proposition 8.2) and equal to $R$.

In general, I cannot prove an existence theorem for a simple algebra structure inducing any given extended annihilation algebra; but the above argument guarantees uniqueness, and explicitly constructs $R$ whenever it exists. In general one can expect to find problems in showing $\bar{R}$ is finitely generated, and proving axiom (S0) for the $\lambda$-bracket defined in (9.3). But I expect $\bar{R}$ always to be free of finite rank, and the $m$ th product to satisfy the local nilpotency axiom. This would give a complete positive classification of all simple $n$-conformal algebras. 


\section{Bibliography}

[BKV] B.Bakalov, V.G.Kac and A.A.Voronov, Cohomology of conformal algebras, preprint

[BPZ] A.A.Belavin, A.M. Polyakov and A.B. Zamolodchikov, Infinite conformal symmetry in two-dimensional quantum field theory, Nucl.Phys. B 241 (1984) $333-380$

[B] R.J.Blattner, A theorem of Cartan and Guillemin, J. Diff. Geom. 5 (1970) 295-305

[Bo] R.Borcherds, Vertex algebras, Kac-Moody algebras, and the Monster, Proc. Natl. Acad. Sci. USA, 83 (1986), 3068-3071

[CK] S.-J.Cheng and V.G.Kac, Conformal modules, Asian J.Math. 1 (1997) 181193

[CKW] S.-J.Cheng, V.G.Kac and M.Wakimoto, Extensions of conformal modules, in "Topological field theory, primitive forms and related topics", Proc. of Taniguchi and RIMS symposia, Progress in Math., Birkhäuser

[DK] A.D'Andrea, V.G.Kac, Structure theory of finite conformal algebra, Selecta Mathematica, to appear.

[GK] I.M.Gelfand and A.A.Kirillov, Sur les corps liés aux algèbres enveloppantes des algèbres de Lie, Publ. Math. IHES 5-19 (1966)

[G] V.Guillemin, A Jordan-Hölder decomposition for a certain class of infinite dimensional Lie algebras, J. Diff. Geom. 2 (1968) 313-345 
[G1] V.Guillemin, Infinite-dimensional primitive Lie algebras J. Diff. Geom. 4 (1970) $257-282$

[K] V.G.Kac, Vertex algebras for beginners, Univ. Lect. Series 10, AMS (1996). Second edition 1998

[K1] V.G.Kac, Simple irreducible graded Lie algebras of finite growth, Math.USSR-Izvestija 2 (1968) 1271-1311

[K2] V.G.Kac, Infinite-dimensional Lie algebras, Bikhäuser, 1983

[K3] V.G.Kac, Formal distribution algebras and conformal algebras, Brisbane Congress in Math.Phys., July 1997

[KW] V.G.Kac and M.Wakimoto, Abelian extensions of the Virasoro conformal algebra, in preparation

[M1] O.Mathieu, Sur un problème de V.G.Kac: la classification de certaines algèbres de Lie graduèes simples, J. Algebra 102, 505-536 (1986)

[M2] O.Mathieu, Classification des algèbres de Lie graduèes simples de croissance $\leq 1$, Invent. Math. 86, 371-426 (1986)

[M3] O.Mathieu, Classification of simple graded Lie algebras of finite growth; Invent. Math. 108, 455-519 (1992)

[NW] W.Nichols and B.Weisfeiler, Differential formal groups of J.F.Ritt, Amer. J. Math 104, no. 5, 943-1003 (1982)

[S] J.-P. Serre, Lie algebras and Lie groups, Benjamin, 1965 